\title{
Functional classification and validation of yeast prenylation motifs using machine learning and genetic reporters
}

Short Title: Prediction of prenylation motifs

\section{Authors:}

Brittany M. Berger ${ }^{1}$ - Conceptualization, methodology, investigation, writing - original draft Wayland Yeung ${ }^{1,2}$ - Conceptualization, methodology, investigation, writing - original draft Arnav Goyal ${ }^{1}$ - Investigation, writing - review \& editing

Zhongliang Zhou ${ }^{3}$ - Investigation, writing - review \& editing

Emily Hildebrandt ${ }^{1}$ - Resources, writing - review \& editing

Natarajan Kannan ${ }^{1,2}$ - Conceptualization, supervision, writing - review \& editing

Walter K. Schmidt ${ }^{1}$ - Conceptualization, supervision, writing - review \& editing

${ }^{1}$ Department of Biochemistry and Molecular Biology, University of Georgia, Athens GA 30602

${ }^{2}$ Institute of Bioinformatics, University of Georgia, Athens GA 30602

${ }^{3}$ Department of Computer Science, University of Georgia, Athens GA 30602

Key words: Prenylation, CaaX, machine learning, FTase

Corresponding author: Walter K. Schmidt, 706-583-8241 (phone), 706-542-1738 (fax), wschmidt@uga.edu (email), 120 Green Street, Athens, GA 30602

Abbreviations: FTase, farnesyltransferase; GGTase-I, geranylgeranyltransferase-I; PrePS, Prenylation Prediction Suite; FPB, FlexPepBind; SVM, support vector machine; SGD, Saccharomyces Genome Database; GBDT, Gradient Boosting Decision Tree 


\section{Abstract}

Protein prenylation by farnesyltransferase (FTase) is often described as the targeting of a cysteine-containing motif (CaaX) that is enriched for aliphatic amino acids at the $\mathrm{a}_{1}$ and $\mathrm{a}_{2}$ positions, while quite flexible at the $\mathrm{X}$ position. Prenylation prediction methods often rely on these features despite emerging evidence that FTase has broader target specificity than previously considered. Using a machine learning approach and training sets based on canonical (prenylated, proteolyzed, and carboxymethylated) and recently identified shunted motifs (prenylation only), this study aims to improve prenylation predictions with the goal of determining the full scope of prenylation potential among the 8000 possible Cxxx sequence combinations. Further, this study aims to subdivide the prenylated sequences as either shunted (i.e., uncleaved) or cleaved (i.e., canonical). Predictions were determined for Saccharomyces cerevisiae FTase and compared to results derived using currently available prenylation prediction methods. In silico predictions were further evaluated using in vivo methods coupled to two yeast reporters, the yeast mating pheromone a-factor and Hsp40 Ydj1p, that represent proteins with canonical and shunted CaaX motifs, respectively. Our machine learning based approach expands the repertoire of predicted FTase targets and provides a framework for functional classification. 


\section{Introduction}

CaaX-type protein prenylation refers to the covalent linkage of a farnesyl or geranylgeranyl isoprenoid group (C15 and $\mathrm{C} 20$, respectively) to proteins containing a $\mathrm{COOH}$-terminal CaaX motif, where $C$ is an invariant cysteine, $a_{1}$ and $a_{2}$ are typically aliphatic residues, and $X$ is one of many amino acids (1). Farnesyltransferase (FTase) and geranylgeranyltransferase-I (GGTase-I) facilitate the isoprenoid addition to the CaaX cysteine thiol, with GGTase-I targeting the subset of CaaX sequences having Leu, Phe or Met at the X position (2-4). For many CaaX proteins, initial isoprenylation is followed by proteolysis that removes the aaX tripeptide, mediated by Rce1p or Ste24p, and carboxymethylation of the isoprenylated cysteine, mediated by isoprenylcysteine carboxyl methyltransferase (ICMT; Ste14p in yeast) (5). These modifications increase the overall $\mathrm{COOH}$-terminal hydrophobicity of modified proteins and often occur to CaaX proteins well-known to be membrane associated (e.g., Ras GTPases).

Despite FTase arguably being the most well characterized enzyme in the CaaX modification pathway, its specificity still remains unclear. Early primary sequence comparisons of known FTase targets often outlined the standard, aliphatic-enriched consensus motif termed CaaX. One of the first methods to predict FTase substrates was developed into the Prenylation Prediction Suite (PrePS) (6). This method evaluated the last 15 amino acids of known prenylated targets, including many Ras and Ras-related GTPases and a few non-canonical sequences for which evidence of prenylation was previously established, to determine a consensus of physio-biochemical properties important for prenylation, which was then used to predict prenylation. PrePS was then applied to create a database of all prenylation predictions across all known proteins, regardless of species (7). The prenylation potential of nearly all 8000 possible CaaX sequences has also been investigated using genetics and high throughput NextGen Sequencing (NGS) in the context of a mutated form of H-Ras (Ras61) that was heterologously expressed in yeast (8). The identified target sequences were consistent with the 
initially described consensus CaaX motif. Parallel in vitro and in silico studies have suggested, however, that FTase may be able to accommodate substantially broader substrates than initially proposed (9-13). A broader consensus for human FTase was also proposed using FlexPepBind (FPB), an approach involving structure-based molecular docking and energy minimization constraints (12). This approach identified several sequences that were not initially expected to be prenylated but subsequently biochemically validated as FTase targets. Despite these new experimental observations and advancements in prenylation prediction methods, many prenylated sequences still fail to be accurately predicted as FTase substrates. Past approaches involving in vitro peptide libraries and metabolic labeling with farnesyl analogs suitable for clickchemistry have been able to identify additional non-canonical sequences as FTase targets, however, peptide libraries are often costly and can be labor intensive and metabolic labeling is limited to cell specific sequences $(9,10,14-17)$. Thus, limitations still prevent exploration of the full scope of prenylation for all 8000 Cxxx sequences.

While the specificity of FTase is emerging to be more flexible than anticipated, the CaaX proteases that mediate subsequent cleavage of the aaX tripeptide appear more stringent, requiring aliphatic residues at $\mathrm{a}_{1}$ and/or $\mathrm{a}_{2}$ positions (18). This observation identifies an inherent bias in many FTase assays due to the use of canonical reporters such as Ras and a-factor where the specificity of the downstream proteases may limit the prenylatable sequences that can be identified. To overcome this bias, we recently developed S. cerevisiae Hsp40 Ydj1p into a novel in vivo reporter for yeast FTase activity (19). Unlike canonical reporters previously used in vivo, the non-canonical CaaX sequence of $\mathrm{Ydj} 1 \mathrm{p}(\mathrm{CASQ})$ is farnesylated, then "shunted" out of the canonical CaaX pathway without being further proteolyzed and carboxymethylated.

Previous studies have shown that yeast require Ydj1p prenylation for growth at high temperatures (i.e., thermotolerance), as evident by a reduced thermotolerant phenotype observed when canonical modification occurs (i.e., prenylation, proteolysis and 
carboxymethylation), and a further reduction in thermotolerance with lack of prenylation (19, 20). This thermotolerant phenotype was used to identify 153 sequences that supported Ydj1p prenylation-dependent yeast growth at high temperatures (21). The recovered sequences were vastly different than standard canonical CaaX sequences, lacking characteristic aliphatic amino acids but consistent with specificities observed through in vitro and in silico studies. For clarity, all 8000 sequences are referred to as Cxxx sequences in this study, while predicted prenylated sequences are referred to as CaaX motifs with qualifiers added to specify those that are canonically modified (i.e., cleaved) or shunted (i.e., uncleaved).

In this study, we used machine learning and yeast genetic data derived from both Ras61 and Ydj1p in vivo reporters to develop methods for predicting the prenylation potential of all 8000 Cxxx sequences within the yeast system. Predictions were then compared to those derived using PrePS, FPB, and Freq. The latter is a frequency-based, in-house method developed in our previous study of Cxxx sequences that support Ydj1p-dependent thermotolerance. Our findings suggest that the use of machine learning with data derived from both canonical and non-canonical reporters results in improved prediction of yeast FTase targets. This approach was also used to develop a first-ever prediction for CaaX proteolysis, leading to effective predictions for establishing whether a prenylated sequence follows the canonical or shunted pathway (i.e., cleaved vs. uncleaved).

\section{Materials and Methods}

\section{Training set curation}

Prenylation: Training sets can be found in Supplemental File S1 and were derived from previously published datasets. The positive set initially included 369 sequences identified through a Ras61 prenylation screen (enrichment score $>3$ at $37^{\circ} \mathrm{C} ; \geq 5$ occurrences) and 153 
sequences identified through a Ydj1p prenylation screen $(8,21)$. The positive training set was curated to form a reduced set of 489 unique sequences by removing duplicate sequences that overlapped between the sets $(n=8)$, sequences found naturally in the Saccharomyces cerevisiae proteome $(\mathrm{n}=21)$, and sequences that had previously been incorporated into reporters $(n=4)$. The negative set initially consisted of 514 sequences that were lowest scoring in the Ras61 prenylation screen (enrichment score $\leq 0.036$ at $37^{\circ} \mathrm{C}$; $\geq 5$ occurrences at $25^{\circ} \mathrm{C}$ ). The negative set was curated to form a reduced set of 508 unique sequences by removing 6 sequences found naturally in the Saccharomyces cerevisiae proteome.

Cleavage: Training sets can be found in Supplemental File S1 and were derived from previously published datasets $(8,21)$. The positive set initially included 153 top scoring Ras 61 sequences (enrichment score $>3$ at $37^{\circ} \mathrm{C} ; \geq 5$ occurrences). From this, the positive training set was reduced to a unique set of 140 by removing duplicate sequences that overlapped with the Ydj1p set $(n=2)$, sequences found naturally in the Saccharomyces cerevisiae proteome $(n=8)$, and sequences that had previously been incorporated into reporters $(n=3)$. The negative set initially included 153 sequences recovered in theYdj1p screen. The negative set was reduced to 136 sequences by removing sequences that were genetically confirmed to be canonically modified $(n=15)$, sequences found naturally in the Saccharomyces cerevisiae proteome $(n=1)$, and sequences that had previously been incorporated into reporters $(n=1)$.

\section{Feature generation \& pre-processing}

Feature generation: In order to generate features for machine learning, we explored three different ways of representing Cxxx sequences: 1) the specific amino acid sequence represented by one-hot encoding, 2) the physico-biochemical features retrieved from the 
AAindex database ( ftp://ftp.genome.jp/pub/db/community/aaindex/; downloaded 1/17/2021) (31), and 3) sequence embedding generated by ESM-1b (https://github.com/facebookresearch/esm; downloaded 2/9/2021), a state-of-the-art Transformer model that was pre-trained on roughly $250 \mathrm{M}$ protein sequences (32). Sequence features were represented by an array of size 60 , which accounts for one-hot encoding of 20 amino acid residues at the 3 variable " $x$ " positions of the Cxxx sequence. AAindex features were represented by an array of size 1659, which accounts for all 553 physico-biochemical features defined by the database for each of the 3 positions. These features were normalized to a range of 0 to 1 in order to equalize their scales. ESM-1b features were generated by taking advantage of the model's ability to account for contextual information, capturing the potential effects of neighboring residues. We represented the $\mathrm{COOH}$-terminal localization of the $\mathrm{Cxxx}$ sequence by front-padding with 100 unspecified "x" residues. In addition, the model added two special characters to represent the beginning and end of the amino acid sequence. This sequence was used to generate an embedding of size $(1280,106)$, which represents a 1280-dimensional abstract description of 104 residue positions plus two special symbols. ESM-1b features were extracted from this embedding by retrieving the positions corresponding with the Cxxx sequence and end-of-sequence character, which resulted in an array of size $(1280,5)$, flattened to size 6400. We retained the positional encoding corresponding to the invariant cysteine due to the model's unique ability to capture contextual information.

Dimensionality reduction: Redundant features were removed through principal component analysis, a standard dimensionality reduction technique (33). This resulted in the reduction of sequence features from 60 to 53 dimensions, AAindex features from 1659 to 50 dimensions, and ESM-1b features from 6400 to 276 dimensions. These reduced features captured $99 \%$ of total variance in each feature set. 


\section{Prediction of Cxxx prenylation \& cleavage}

Scoring: We quantified the performance of all prediction models based on accuracy, precision, recall, and F1-score. Reported values indicate the mean across 10 -fold cross validation while confidence intervals indicate the standard deviation.

Position-specific scoring matrix (PSSM): We constructed a PSSM based on prenylated or cleaved motifs. The amino acid distribution was normalized against a background amino acid distribution defined by the BLOSUM62 substitution matrix (34) with a pseudo-count of 0.05 . The resulting model was used to calculate the log probability of a given sequence being prenylated or cleaved. In order to obtain binary predictions, we defined a cutoff log probability that best separated the positive from the negative examples.

Machine learning algorithms: We tested the performance of various machine learning algorithms as implemented by Scikit-learn (35). The parameters of individual predictors were optimized by grid search. Specific algorithms tested were support vector machine (SVM), Naïve Bayes, knearest neighbor (kNN), and Gradient Boosted Decision Tree (GBDT). In subsequent analyses, we estimated the probabilities of each prediction for SVM through Platt scaling (23).

Software: All computational analyses, unless otherwise mentioned, were implemented in Python 3 using NumPy (36) and PyTorch (37). Figure plots were created using Matplotlib (38), seaborn (39), WebLogo3(40), and Adobe Illustrator. For WebLogo3, a custom color scheme was used where cysteine $(C)$ was blue, polar charged amino acids $(H, K, R, E, D)$ were green, polar uncharged amino acids (N, Q, S, T, Y) were black, branched-chain amino acids $(\mathrm{L}, \mathrm{I}, \mathrm{V})$ were red, and all other amino acids ( $F, A, P, G, M, W)$ were purple. This scheme matches that used in a previously published study of FTase specificity by our group (21). 
Cut-offs used for predictions by prenylation methods: For analysis with the Prenylation Prediction Suite, (PrePS; https://mendel.imp.ac.at/PrePS), all 8000 Cxxx sequences were evaluated in the context of human H-Ras (RQHKLRKLNPPDESGPGCMSCKCxxx). While PrePS only requires 15 amino acids for scoring, 26 were used to remain consistent with previous studies $(19,21)$. For PrePS, sequences scoring greater than -2 were deemed positive predictions. For FlexPepBind, sequences scoring greater than -1.1 were deemed positive predictions, consistent with the stringent threshold defined by the original study (12). For Freq, prenylation sequences scoring greater than -1 were deemed positive predictions, while sequences scoring greater than 0 were deemed positive predictions for cleavage (21).

\section{Experimental validation}

Yeast strains: Strains used in this study are listed in Supplementary Materials Table S3. Lithium acetate-based transformation methods were used to introduce plasmids into yeast strains (21, 41). All strains were propagated at $25^{\circ} \mathrm{C}$ unless otherwise stated, in YPD or appropriate selection media. For yWS2393, deletion of STE24 was carried out in strain yWS44 (mfa1A mfa2A) using: a DNA fragment from pWS405 (CEN URA3 ste24::KanMX4) that was transformed into yWS44 (42). G418 resistant colonies were checked by PCR for integration of ste24::KANMX4 at the STE24 locus. For yWS2462, deletion of RCE1 was carried out in strain yWS44 using a rce $1:: K A N$ fragment recovered by PCR from the haploid yeast gene deletion collection (43), and integration at the RCE1 locus was confirmed by PCR.

Plasmids: Plasmids used in this study are listed in Supplementary Material Table S4. All plasmids newly created for this study were constructed using methods previously reported (19, $21,44)$. Briefly, new plasmids encoding Ydj1p or a-factor reporters were constructed using PCR-directed recombination. Mutagenic oligonucleotides (Table S5) encoding desired Cxxx 
sequences were co-transformed with linearized or gapped parent plasmids, transformation mixes plated onto appropriate selection media, and plasmids recovered from surviving colonies. Plasmids were sequenced through the entire open reading frame of the reporter using an appropriate DNA sequencing primer and a sequencing service (Genewiz, Southfield NJ; Eurofins Genomics, Louisville, Kentucky). pWS130 (2 $\mu$ URA3 $\left.P_{P G K}-H s R c e 1 \Delta 22\right)$ was constructed by subcloning a PCR-derived fragment from a baculovirus expression vector encoding HsRce1 122 (courtesy of P. Casey, Duke University). The PCR fragment was designed to contain $5^{\prime}$ BamHI and 3' Pstl sites that were used for subcloning, where the latter was blunted with T4 Polymerase prior to cloning into the BamHI and Sacll sites of pWS28 $(2 \mu$ URA3 PPGK) (45). pWS1609 was created from pWS1275 (2 $\mu$ URA3 $\left.P_{P G K}-H A-H s S T E 24\right)$ by PCR-directed, plasmid-based recombination to eliminate the HA-tag, followed by subcloning $\mathrm{P}_{\mathrm{PGK}}-$ HsSTE24 into pRS316 (CEN URA3) $(44,46)$.

Ydj1p gel shift assay: The prenylation status of Ydj1p was examined as described previously. Briefly, yeast strains expressing Ydj1p were cultured to $A_{600} 0.9-1.1$ at $30{ }^{\circ} \mathrm{C}$ in synthetic complete media lacking uracil (SC-U). Cell pellets of the same mass were collected by centrifugation, washed with water, and cell extracts prepared by alkaline hydrolysis followed by TCA precipitation (47). Cell extracts were resuspended in Sample Buffer (250 mM Tris, 6 M Urea, $5 \% \beta$-mercaptoethanol, 4\% SDS, 0.01\% bromophenol blue, $\mathrm{pH} 8$ ) and analyzed by SDSPAGE and immunoblotting with rabbit anti-Ydj1p antibody (courtesy of Dr. Avrom Caplan) and HRP conjugate antibody in TBST (10 mM Tris, $150 \mathrm{mM} \mathrm{NaCl,} \mathrm{0.1 \%} \mathrm{Tween-20;} \mathrm{pH} \mathrm{7.5)} \mathrm{with} \mathrm{1 \%}$ milk/TBST. Blots were developed with WesternBright ECL Spray (Advansta Inc, San Jose, California), and images captured using X-ray film or a digital imager (Kwikquant, Kindle Biosciences, Greenwich, Connecticut). 
Yeast mating assay: Mating assays were performed as previously described (21). Briefly, MATa and MATa strains were cultured to saturation at $30^{\circ} \mathrm{C}$ in synthetic complete media lacking leucine (SC-L) and YPD, respectively, then normalized to an $A_{600}$ value of 1 by dilution with appropriate sterile media. MATa cultures were mixed individually 1:10 with the MATa cultures, each mixture was serially diluted 10 -fold using the normalized MATa culture as the diluent, and serial dilutions were pinned onto minimal (SD) and synthetic complete media lacking lysine plates (SC-K). Plates were incubated for 72 hours and imaged against a black background using flat-bed scanner. Images were adjusted using Photoshop to optimize the dynamic range of signal by adjusting input levels to a fixed range of 25-150.

\section{Data Availability}

Strains and plasmids are available upon request. All relevant datasets for this study are included in the supplemental files of manuscript. The coding used is publicly available at the GitHub repository: https://github.com/waylandy/prenylation cleavage prediction.

\section{Results}

Prenylated and cleaved Cxxx sequences can be distinguished based on primary amino acid

\section{sequence feature}

To evaluate whether the information encoded in primary sequences can be used to distinguish prenylated and cleaved sequences, we first curated a training dataset from two previously published genetic screens that used Ras61 and Ydj1p as reporters $(8,21)$. As prenylation is necessary for the optimal function of both Ras61 and Ydj1p reporter activities, we curated 489 prenylated sequences by combining the top performing sequences from both screens. Another 508 low performing sequences from the Ras61 study served as the non-prenylated set; the Ydj1p-based study did not yield information for low-performing sequences. Notably, prenylation 
and proteolysis have historically been considered coupled events, and as such, previous methodologies do not report on proteolysis. However, the Ydj1p reporter is uniquely able to differentiate between shunted (i.e., only prenylated) and cleaved sequences (i.e., canonically modified; prenylated, cleaved and carboxymethylated). Thus, we curated 136 sequences from the Ydj1p screen and 140 sequences from the Ras61 screen to serve as shunted and cleaved sets, respectively (21).

We next evaluated the contribution of three sequence representation methods: one hot encoding of primary sequence (sequence-only), AAindex, and ESM-1b. These methods capture different aspects of Cxxx sequences (see Materials \& Methods for additional details) in classifying prenylated and non-prenylated sequences. Two-dimensional projections of each set of features revealed that sequence-only and AAindex features readily distinguish prenylated and non-prenylated sequences, while ESM-1b exhibited poor separation (Fig 1A). As AAindex appeared to best separate the prenylated and non-prenylated sequences, we used Weblogo to analyze the sequences clustered with the right and left sides of the projection (Fig 1B). The right-side cluster was mostly composed of prenylated sequences that closely resembled the canonical definition of CaaX, with a clear enrichment of aliphatic amino acids at the $\mathrm{a}_{2}$ position, and to some extent the $\mathrm{a}_{1}$ position. By comparison, the left-side cluster was a mixed population of prenylated and non-prenylated sequences lacking these canonical aliphatic residues. Although ESM-1b encodes more information (276 dimensions to capture $99 \%$ variance in data compared to 50 dimensions for sequence and AAindex (see Materials \& Methods)), the poor separation observed with ESM-1b is likely a consequence of the additional contextual information which could not be sufficiently compressed into two-dimensional space. All three sequence representation methods, meanwhile, are suitable for separating cleaved and uncleaved sequences (Fig 1C). 


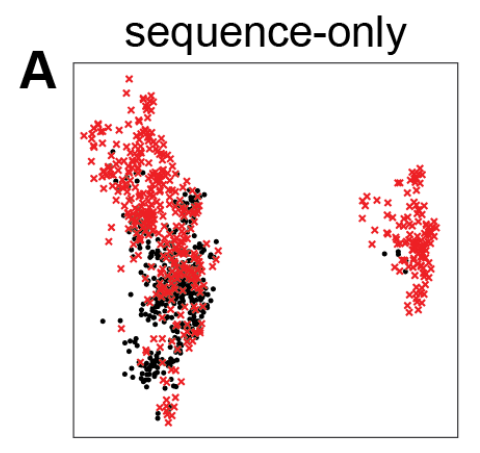

B
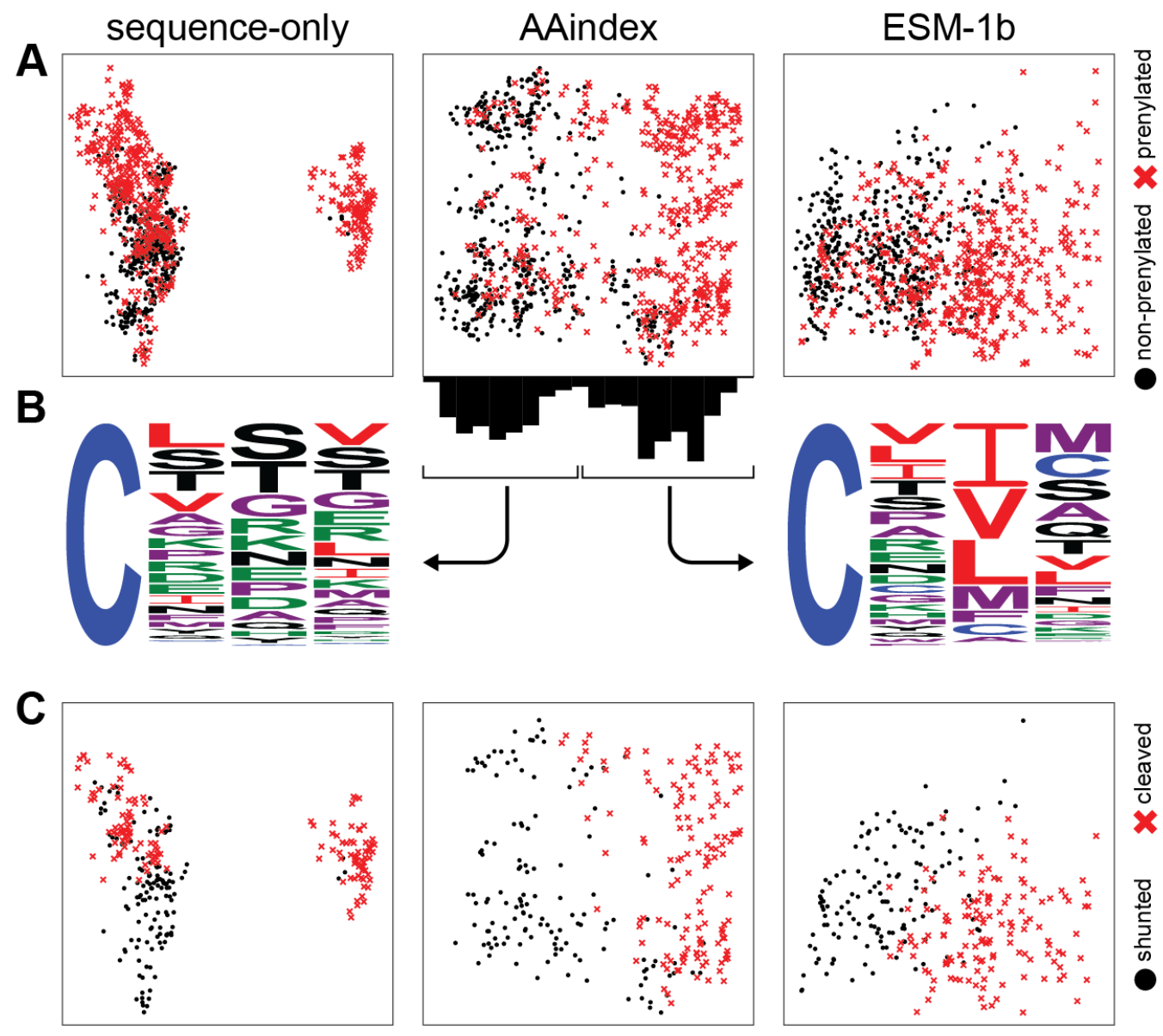

Fig 1. Separation of sequences by machine learning-based methods. A) Data points from all three features sets: sequence only, AAindex and ESM-1b, are represented as a two-dimensional projection of prenylated (red $\mathrm{x}$ ) and non-prenylated sequences (black dot). The axes are not shown as they represent a linear combination of all features that maximizes variance. B) Bimodal distribution of sequences across the $\mathrm{X}$-axis from the AAindex manifold were graphed as sequence logos. The distribution shown on the left contains a mix of non-prenylated Cxxx sequences and prenylated, non-canonical sequences, while the one on the right mostly consists of prenylated, canonical CaaX sequences. C) A similar two-dimensional projection was used to represent cleaved (red $\mathrm{x}$ ) and shunted (i.e., uncleaved) sequences (black dot).

SVM-ESM-1b outperforms several machine learning based models for prenylation and cleavage predictions

A position-specific scoring matrix model (PSSM) is a common bioinformatics method employed for motif detection (22). A variation of this method is used by the PrePS model (6). We thus constructed a PSSM model based on the Cxxx sequences from our curated datasets to 
establish a baseline for comparisons of other prenylation and cleavage prediction models. The PSSM model applied to a curated dataset of both canonical and non-canonical sequences achieved $83.8 \pm 3.3 \%$ accuracy for prenylation predictions, and a second PSSM model to predict cleavage achieved $93.8 \pm 4.6 \%$ accuracy, based on 10 -fold cross validation (Table 1 )

We next evaluated whether the baseline PSSM classification accuracy could be improved through different representations of Cxxx sequences using machine learning (see Materials \& Methods for details on methods used).

Table 1. Performance of various models for prenylation prediction.

\begin{tabular}{|c|c|c|c|c|c|c|}
\hline Modela & Features $^{b}$ & Accuracyc & Precision & Recall & F1 & Validation \\
\hline PSSM & sequence & $83.8 \pm 3.3$ & $87.7 \pm 3.5$ & $77.9 \pm 5.9$ & $82.4 \pm 3.8$ & $68.4(13 / 19)$ \\
\hline SVM & sequence & $86.0 \pm 2.7$ & $86.5 \pm 4.0$ & $84.9 \pm 3.8$ & $85.6 \pm 2.8$ & $84.2(16 / 19)$ \\
\hline SVM & AAindex & $85.1 \pm 3.5$ & $86.6 \pm 4.1$ & $82.4 \pm 3.6$ & $84.4 \pm 3.6$ & $73.7(14 / 19)$ \\
\hline SVM & ESM-1b & $86.4 \pm 3.0$ & $86.6 \pm 3.3$ & $85.5 \pm 4.1$ & $86.0 \pm 3.1$ & $84.2(16 / 19)$ \\
\hline GBDT & sequence & $86.2 \pm 2.4$ & $87.9 \pm 3.5$ & $83.4 \pm 3.5$ & $85.5 \pm 2.6$ & $68.4(13 / 19)$ \\
\hline GBDT & AAindex & $86.2 \pm 2.8$ & $87.2 \pm 3.5$ & $84.3 \pm 4.3$ & $85.6 \pm 3.0$ & 73.7 (14/19) \\
\hline GBDT & ESM-1b & $85.0 \pm 2.9$ & $85.8 \pm 3.8$ & $83.2 \pm 3.6$ & $84.4 \pm 3.0$ & $78.9(15 / 19)$ \\
\hline $\begin{array}{l}\text { Näive } \\
\text { Bayes }\end{array}$ & sequence & $82.9 \pm 1.8$ & $85.5 \pm 3.1$ & $78.7 \pm 3.3$ & $81.9 \pm 1.9$ & $63.2(12 / 19)$ \\
\hline $\begin{array}{l}\text { Näive } \\
\text { Bayes }\end{array}$ & AAindex & $82.1 \pm 3.0$ & $82.2 \pm 4.0$ & $81.4 \pm 3.6$ & $81.7 \pm 3.0$ & $73.7(14 / 19)$ \\
\hline $\begin{array}{l}\text { Näive } \\
\text { Bayes }\end{array}$ & ESM-1b & $73.2 \pm 2.3$ & $70.4 \pm 1.9$ & $78.3 \pm 3.9$ & $74.1 \pm 2.5$ & $57.9(11 / 19)$ \\
\hline kNN & sequence & $84.1 \pm 3.7$ & $82.7 \pm 4.3$ & $85.5 \pm 4.3$ & $84.0 \pm 3.7$ & $78.9(15 / 19)$ \\
\hline kNN & AAindex & $82.7 \pm 2.3$ & $83.5 \pm 3.1$ & $81.0 \pm 3.0$ & $82.2 \pm 2.3$ & $78.9(15 / 19)$ \\
\hline kNN & ESM-1b & $83.0 \pm 2.3$ & $82.4 \pm 3.5$ & $83.4 \pm 2.4$ & $82.9 \pm 2.1$ & $78.9(15 / 19)$ \\
\hline \multicolumn{7}{|c|}{ 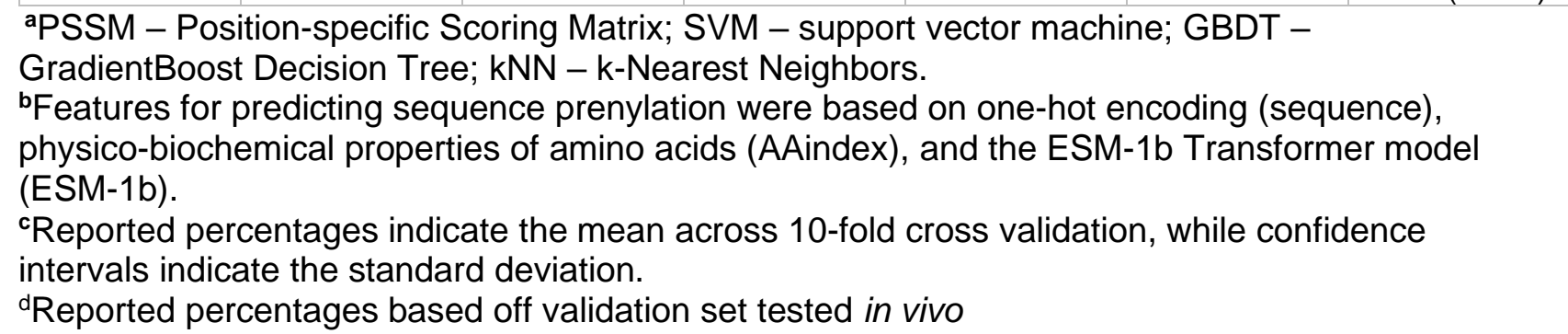 } \\
\hline
\end{tabular}

For prenylation, most of the 12 machine learning methods evaluated scored above $80 \%$ in all categories. We selected the best model based on F1-score, defined as the harmonic mean of 
precision and recall. Based on this criterion, support vector machine (SVM) paired with ESM-1b features was the best overall performer. We next evaluated how well each model predicted prenylation of a validation set of $31 \mathrm{Cxxx}$ sequences that were not part of training sets (Table S1). Within this validation set, 19 of the 31 sequences naturally occur in the yeast proteome. The reasons for choosing these 19 sequences varied: 12 formed 6 pairs that differ by only one amino acid, for example Ras2 (CIIS) and Hmg1 (CIKS); 7 exhibited varying predictions for prenylation with multiple prediction methods (e.g., PrePS, Freq, etc.). The remaining 12 sequences were chosen due to differing predictions by SVM-ESM-1b, PrePS, and the frequency-based scoring system (Freq). The sequences representing the validation set were incorporated onto Ydj1p and prenylation evaluated by a gel shift assay (Fig 4A, Table 3), with the exception of one sequence (CQSQ) that had been previously evaluated (21). Relative to PSSM, most machine learning methods improved at predicting actual prenylation (Table 1; Validation score). SVM was repeatedly the best overall performer when paired with ESM-1b features. Considering the results of performance testing with training and naïve test sets, SVM paired with ESM-1b features was chosen as the preferred machine learning method for additional prenylation prediction studies.

We also explored sequence cleavage using similar methods (Table 2). All models performed comparably well based on 10 -fold cross validation, with most scoring above $90 \%$ in all categories. As observed for prenylation prediction, many of the models surpassed the PSSM model for accuracy and recall, and only 1 bettered PSSM for precision (Table 2). Overall, SVM paired with either sequence or ESM-1b features achieved the best F1-score for predicting cleavage. As SVM-ESM-1b had the smaller standard deviation, it was chosen as the preferred method for cleavage prediction. We next evaluated how well each model predicted cleavage of the validation set of 19 naturally occurring Cxxx sequences. We incorporated these 19 sequences onto the a-factor reporter that conditionally requires both prenylation and cleavage 
for bioactivity (Fig 4B). Because 5 of the sequences were not observed to be prenylated by gelshift assay (CIKS, CIDL, CSEI, CSGL, CSGK), these sequences were not expected to exhibit any a-factor activity, which was indeed the case. For this reason, these 5 sequences were not included statistically in the a-factor validation set. The remaining 14 sequences either possessed a-factor activity, indicative of cleavage, or lacked bioactivity, indicative of only being prenylated. Surprisingly, we found that several models outperformed SVM-ESM-1b on the validation set when considering the 14 prenylated sequences (Table 3, Table S1). We caution, however, that the small size of the validation set may lack sufficient statistical power to make proper comparisons and conclusions.

Table 2. Performance of various models for cleavage prediction.

\begin{tabular}{|c|c|c|c|c|c|c|}
\hline Model $^{\mathbf{a}}$ & Features & Accuracy & Precision & Recall & F1 & Validation \\
\hline PSSM & sequence & $93.8 \pm 4.6$ & $97.1 \pm 4.5$ & $90.7 \pm 7.9$ & $93.6 \pm 4.9$ & $89.4(12 / 14)$ \\
\hline SVM & sequence & $97.5 \pm 2.3$ & $96.7 \pm 4.3$ & $98.6 \pm 2.9$ & $97.5 \pm 2.2$ & $78.9(10 / 14)$ \\
\hline SVM & AAindex & $96.4 \pm 2.8$ & $95.3 \pm 4.1$ & $97.9 \pm 3.3$ & $96.5 \pm 2.7$ & $78.9(10 / 14)$ \\
\hline SVM & ESM-1b & $97.5 \pm 1.6$ & $97.3 \pm 3.3$ & $97.9 \pm 3.3$ & $97.5 \pm 1.6$ & $78.9(10 / 14)$ \\
\hline & & & & & & \\
\hline GBDT & sequence & $94.9 \pm 3.4$ & $94.6 \pm 3.9$ & $95.8 \pm 5.7$ & $95.0 \pm 3.4$ & $52.6(8 / 14)$ \\
\hline GBDT & AAindex & $86.9 \pm 3.2$ & $87.7 \pm 4.3$ & $85.3 \pm 3.1$ & $86.4 \pm 3.2$ & $73.7(11 / 14)$ \\
\hline GBDT & ESM-1b & $86.2 \pm 1.9$ & $87.0 \pm 2.8$ & $84.5 \pm 2.3$ & $85.7 \pm 1.9$ & $78.9(10 / 14)$ \\
\hline Näive Bayes & sequence & $89.9 \pm 6.0$ & $89.1 \pm 6.6$ & $91.5 \pm 7.0$ & $90.1 \pm 5.9$ & $68.4(9 / 14)$ \\
\hline Näive Bayes & AAindex & $94.2 \pm 2.4$ & $94.5 \pm 4.0$ & $94.3 \pm 4.3$ & $94.3 \pm 2.3$ & $89.4(12 / 14)$ \\
\hline Näive Bayes & ESM-1b & $85.5 \pm 7.6$ & $85.3 \pm 7.3$ & $86.4 \pm 10.3$ & $85.7 \pm 7.9$ & $68.4(9 / 14)$ \\
\hline & & & & & & \\
\hline kNN & sequence & $94.9 \pm 4.3$ & $96.5 \pm 4.7$ & $93.6 \pm 5.9$ & $94.9 \pm 4.4$ & $84.2(12 / 14)$ \\
\hline kNN & AAindex & $94.6 \pm 3.3$ & $92.4 \pm 5.9$ & $97.9 \pm 3.3$ & $94.9 \pm 3.0$ & $78.9(10 / 14)$ \\
\hline kNN & ESM-1b & $95.3 \pm 4.5$ & $94.7 \pm 5.6$ & $96.4 \pm 5.8$ & $95.4 \pm 4.5$ & $78.9(10 / 14)$ \\
\hline
\end{tabular}

aTerms, definitions, and calculations are as described for Table 1.

Global predictions for prenylation and cleavage of Cxxx sequence space

After evaluating different models for prenylation and cleavage with our curated training and validation sets, we chose SVM paired with ESM-1b to predict both prenylation and cleavage for the full scope of Cxxx sequences (Supplemental File S2). In the case of prenylation, our model was trained to make binary predictions, but these sequence predictions are better represented 
on a continuum as partial prenylation could occur, resulting in sequences with fractions of the protein population being prenylated. In order to model this continuum, we obtained probabilistic outputs for the SVM model by Platt scaling (23) (Fig 2). We note that this method only provides an estimated probability, which does not perfectly translate to a strict cutoff value for the actual binary classification. Altogether, our analysis of all 8000 Cxxx sequences predicts that $67 \%$ $(n=5373)$ are unmodified, $18 \%(n=1420)$ are shunted (i.e., prenylation only), and $15 \%(n=1217)$ cleaved (i.e., canonically modified; prenylated, cleaved, and carboxylmethylated). (Fig 3A,D). We also made global predictions using the SVM-ESM-1b prenylation model paired with our previously published Freq method that outperformed all machine learning models on cleavage validation score (Fig 3B,E), as well as using Freq for both prenylation predictions and cleavage (Fig 3C,F) (21). All predictions were qualitatively similar, with the majority of the 8000 sequences being unmodified, and more shunted sequences predicted relative to canonical sequences.

A

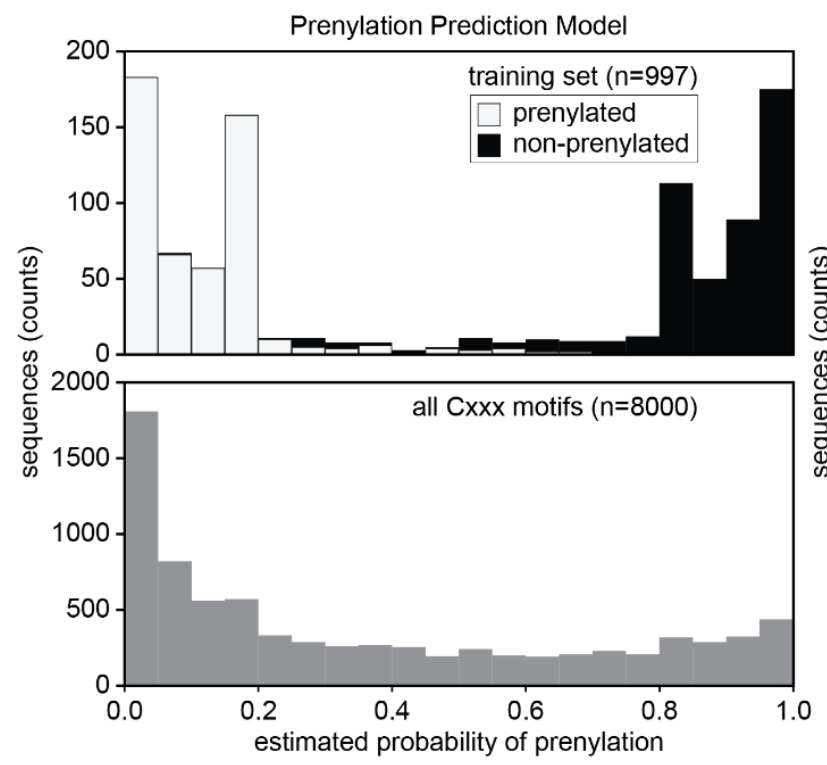

B

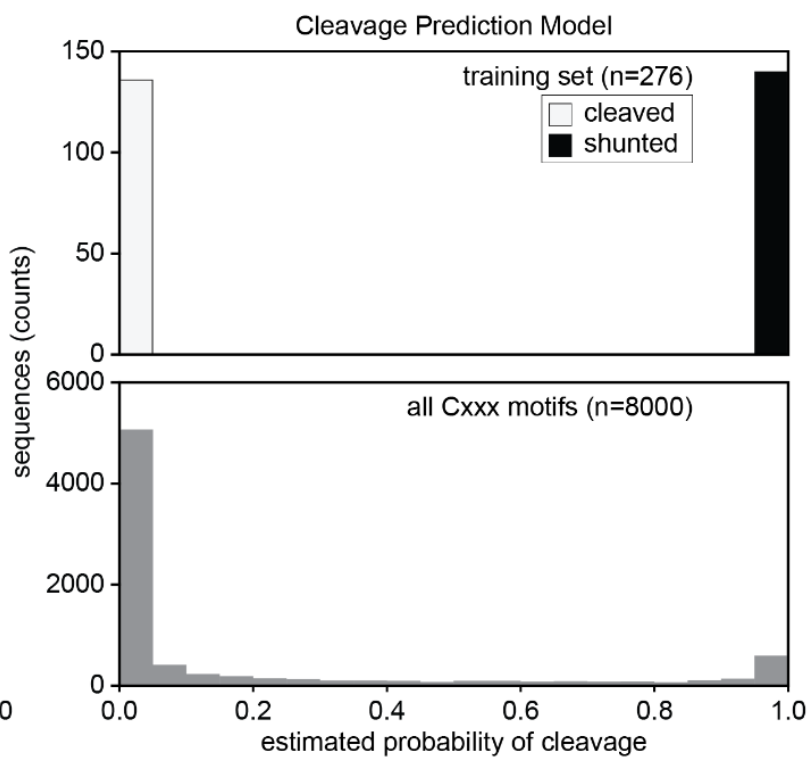

Fig 2. Probability distributions for prenylation and cleavage predictions made by SVM-ESM-1b. Probability distributions for both prenylation $(A)$ and cleavage $(B)$ determined for the training sets (top) and for all $8000 \mathrm{Cxxx}$ motifs (bottom). A) For prenylation, the training set distribution is represented as a stacked bar plot where prenylated sequences are white, while non-prenylated sequences are black. B) For cleavage, the training set distribution is represented as a stacked bar plot where shunted sequences (prenylation only) are black and cleaved sequences for proteolysis. The probability distributions were determined for the training sets (top) and for all 8000 Cxxx motifs (bottom). 

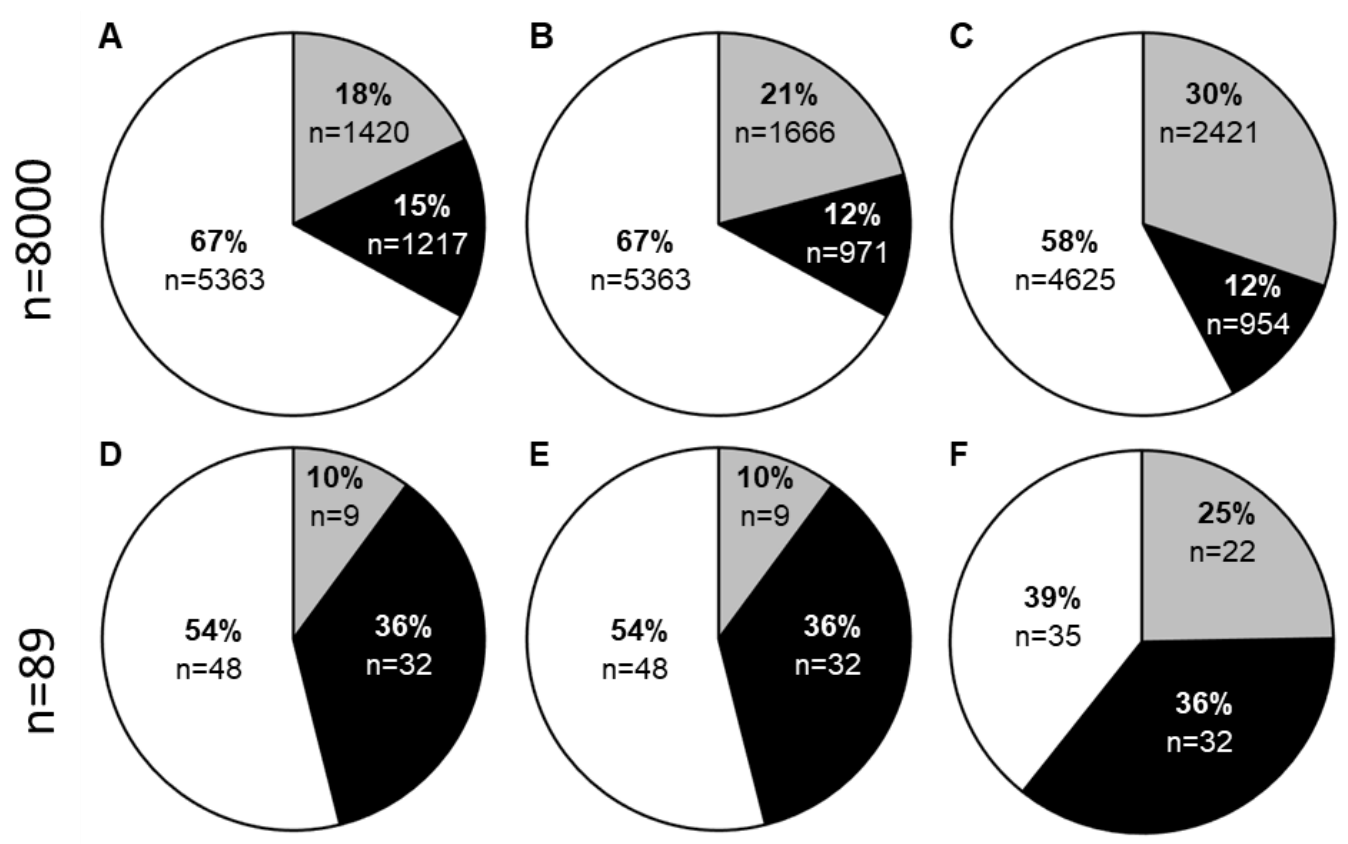

Fig 3. Predictions for modification of Cxxx sequences based on various methods. Predictions for prenylation and cleavage for all $8000 \mathrm{Cxxx}$ sequences (A-C) and 89 naturally occurring yeast $\mathrm{Cxxx}$ sequences $(D-F)$. Models used were SVM-ESM-1b for both predictions (A,D), SVM-ESM-1b for prenylation and Freq for cleavage $(B, E)$, and Freq for both $(C, F)$. Predictions are binned as nonprenylated (white), shunted (gray), and cleaved sequences (black).

$\underline{\text { Comparisons to previous prenylation methods and evaluation of yeast proteome predictions }}$

Several prenylation predictors have been developed previously. These include: PrePS, a PSSM-based model; FlexPepBind (FPB), a molecular docking-based model encompassing energy scores; and Freq, an in-house method developed by scoring the frequency of residues at each position in the positive and negative testing sets used for machine learning in this study. Relative to all 8000 Cxxx sequence space, our SVM-ESM-1b based model predicts prenylation for more sequences (33\%) in comparison to PrePS (20\%) and FlexPepBind (17\%), but less by comparison to Freq (42\%). While Freq predicts more prenylated sequences, it is important to note that this method overpredicts prenylation in the negative training set relative to the SVMESM-1b model ( $\sim 0 \%$ vs. 3\%, respectively). A potential explanation for the higher false positive rate of Freq may be that this method does not explicitly encode contextual information when 
generating features. Overall, we conclude that the SVM-ESM-1b based machine learning model predicts more prenylatable space as compared to PrePS and FlexPepBind, and may more accurately predict prenylation than our previously reported Freq method. Regarding CaaX cleavage prediction, Freq has been the only available method for binning prenylated sequences as either shunted or cleaved. Freq predicts more shunted sequences relative to PSSM-based predictions (30\% vs. $21 \%$, respectively), while the prediction for cleaved sequences is the same in both cases $(12 \%)$.

Altogether, the yeast genome contains 89 proteins having $\mathrm{Cxxx}$ at the $\mathrm{COOH}$-terminus. Prenylation and cleavage predictions were determined for the Cxxx sequences associated with these proteins using our SVM-ESM-1b and PSSM models, respectively. SVM predicted 41 yeast Cxxx proteins to be prenylated, where 32 were canonically modified and 9 were shunted (Fig 3B). While many of the canonically modified CaaX proteins have been previously characterized (a-factor, Ras, etc.), some have non-canonical Cxxx sequences and have not been previously evaluated for their prenylation status, including Cst26p (CFIF; an acyltransferase) and Sua5p (CIQF; involved in threonylcarbamoyladenosine synthesis). Of the 89 Cxxx sequences associated with the yeast proteome, 19 were directly evaluated in this study in the context of the Ydj1p reporter (Table 3, Fig 4A,S1). The SVM-ESM-1b model correctly predicted the prenylation (both positive and negative) for $84 \%$ of the sequences. By comparison, PrePS was next best, correctly predicting $79 \%$, followed by Freq correctly predicting $74 \%$, and FPB correctly predicting 58\%. Because SVM-ESM-1b, PrePS, and Freq performed similarly in predicting prenylation of naturally occurring Cxxx sequences, we evaluated additional sequences to better differentiate the prediction methods. Our lab possesses a large collection of plasmids encoding Ydj1-Cxxx variants ( $n>200)$. Excluding those with Cxxx sequences that were part of machine learning training sets and others for which SVM-ESM-1b and PrePS had the same prediction led us to 12 plasmids with varying differential 
predictions by SVM, PrePS, and Freq. (Table 4). For these 12 Cxxx sequences, Freq correctly predicted 10, SVM-ESM-1b correctly predicted 9, and PrePS correctly predicted 4 (Table 4, Fig 4B). All 12 sequences were prenylated to some degree, resulting in a high percentage of false negatives for PrePS and SVM. Thus, for the combined set of 31 sequences evaluated, SVM correctly predicted $81 \%$ (25/31), Freq correctly identified $77 \%$ (24/31), and PrePS correctly predicted 61\% (19/31) (Table 5).

Table 3. Comparison of prenylation and cleavage prediction models with empirical observations.

\begin{tabular}{|c|c|c|c|c|c|c|c|c|c|c|}
\hline & & & \multicolumn{5}{|c|}{ Prenylation } & \multicolumn{3}{|c|}{ Cleavage } \\
\hline & yeast protein & CaaX & SVM $^{a, b}$ & PrePS & Freq & FPB & Observed ${ }^{c}$ & SVM $^{a}$ & Freq & Observed $^{d}$ \\
\hline \multirow{12}{*}{$\begin{array}{c}\text { similar } \\
\text { sequences }\end{array}$} & Ras2 & CIIS & + & + & + & + & + & + & + & + \\
\hline & Hmg1 & CIKS & - & - & - & - & - & NA & NA & NA \\
\hline & Rho2 & CIIL & + & + & + & - & + & + & + & + \\
\hline & Ssp2 & CIDL & - & - & - & - & - & NA & NA & NA \\
\hline & Skt5, MiY1 & CVIM & + & + & + & - & + & + & + & + \\
\hline & Tbs 1 & CVKM & - & - & - & - & + & + & - & - \\
\hline & YDL022C-A & CSII & + & + & + & + & + & + & + & + \\
\hline & YBR096W & CSEI & - & - & - & - & - & NA & NA & NA \\
\hline & YMR265C & CSNA & - & - & + & - & + & - & - & - \\
\hline & Pet18 & CYNA & - & - & - & + & + & - & - & - \\
\hline & Lih1 & CSGL & - & - & + & - & - & NA & NA & NA \\
\hline & Cup1 & CSGK & - & - & - & - & - & NA & NA & NA \\
\hline \multirow{7}{*}{$\begin{array}{c}\text { other } \\
\text { sequences }\end{array}$} & Nap1 & CKQS & + & + & + & - & + & - & - & - \\
\hline & Cst26 & CFIF & + & + & - & - & + & + & - & - \\
\hline & YIL134C-A & CAPY & + & + & - & - & + & - & - & - \\
\hline & Atr1 & CTVA & + & + & + & + & + & + & + & + \\
\hline & Las21 & CALD & + & - & + & + & + & - & + & - \\
\hline & YDL009C & CAVS & + & + & + & + & + & - & + & + \\
\hline & Sua5 & CIQF & + & + & + & - & + & + & - & - \\
\hline \multicolumn{3}{|c|}{ number observed/predicted } & $16 / 19$ & $15 / 19$ & $14 / 19$ & $11 / 19$ & & $10 / 14$ & $13 / 14$ & \\
\hline
\end{tabular}

aSigns represent predictions of prenylation and cleavage that were reported as positive (+) or negative (-) by the indicated model. NA - not applicable.

bSVM - SVM-ESM-1b; PrePS - Prenylation Prediction Suite; Freq - in-house, frequency-based; FPB - FlexPepBind.

'Observed by Ydj1 prenylation gel shift - see Fig 4A, Fig S1.

dObserved by a-factor mating - see Fig 4C. 
Table 4: Comparison of SVM-ESM-1b and PrePS prenylation predictions with empirical observations.

\begin{tabular}{|c|c|c|c|c|c|}
\hline \multirow{2}{*}{$\begin{array}{c}\text { Reporter } \\
\text { Ydj1-Cxxx }\end{array}$} & \multicolumn{4}{|c|}{ Prenylation } & \multirow[b]{2}{*}{ Observed $^{c}$} \\
\hline & $S_{V} M^{a, b}$ & PrePS & Freq & FPB & \\
\hline CAAQ & + & - & + & - & + \\
\hline CAHQ & + & - & + & - & + \\
\hline CASA & + & - & + & - & + \\
\hline CKQH & + & - & + & - & + \\
\hline CNLI & + & - & + & - & + \\
\hline CSFL & + & - & + & - & + \\
\hline CVAA & + & - & + & - & + \\
\hline CVFM & + & - & + & - & + \\
\hline CKQG & - & + & + & - & + \\
\hline CKQL & - & + & + & - & + \\
\hline CQTS & - & + & - & - & + \\
\hline $\mathrm{CQSQ}^{\mathrm{d}}$ & + & + & - & - & + \\
\hline $\begin{array}{c}\text { number } \\
\text { observed/predicted }\end{array}$ & $9 / 12$ & $4 / 12$ & $10 / 12$ & $0 / 12$ & \\
\hline
\end{tabular}

aSigns represent predictions of prenylation and cleavage that were reported as positive (+) or negative (-) by the indicated model. NA - not applicable.

bSVM - SVM-ESM-1b; PrePS - Prenylation Prediction Suite; Freq - in-house, frequency-based; FPB - FlexPepBind.

'Observed by Ydj1 prenylation gel shift - see Fig 4B.

dObservation previously reported (21).

Table 5: Summary of prenylation and cleavage predictions

\begin{tabular}{|c|c|c|c|c|c|c|}
\hline & \multicolumn{4}{|c|}{ Prenylation } & \multicolumn{2}{|c|}{ Cleavage } \\
\hline & SVM $^{a}$ & PrePS & Freq & FPB & SVM $^{\mathrm{a}}$ & Freq \\
\hline number observed/predicted ${ }^{b}$ & $25 / 31$ & $19 / 31$ & $24 / 31$ & $11 / 31$ & $10 / 14$ & $13 / 14$ \\
\hline \%observed/predicted & $81 \%$ & $61 \%$ & $77 \%$ & $28 \%$ & $71.4 \%$ & $92.9 \%$ \\
\hline number false positive & $0 / 20$ & $0 / 14$ & $1 / 22$ & $0 / 6$ & $3 / 7$ & $1 / 7$ \\
\hline$\%$ false positive & 0 & 0 & $4.5 \%$ & 0 & $42.8 \%$ & $14.3 \%$ \\
\hline number false negative & $6 / 11$ & $12 / 17$ & $5 / 9$ & $20 / 25$ & $1 / 7$ & $0 / 7$ \\
\hline$\%$ false negative & $54.5 \%$ & $70.6 \%$ & $55.5 \%$ & $80 \%$ & $14.3 \%$ & 0 \\
\hline
\end{tabular}

a SVM - SVM-ESM-1b; PrePS - Prenylation Prediction Suite; Freq - in-house, frequencybased; FPB - FlexPepBind.

${ }^{\text {b}}$ Values determined by empirical data via Ydj1 prenylation gel shift (prenylation, Fig 4A,B, Fig S1) or a-factor mating (cleavage, Fig 4C). 

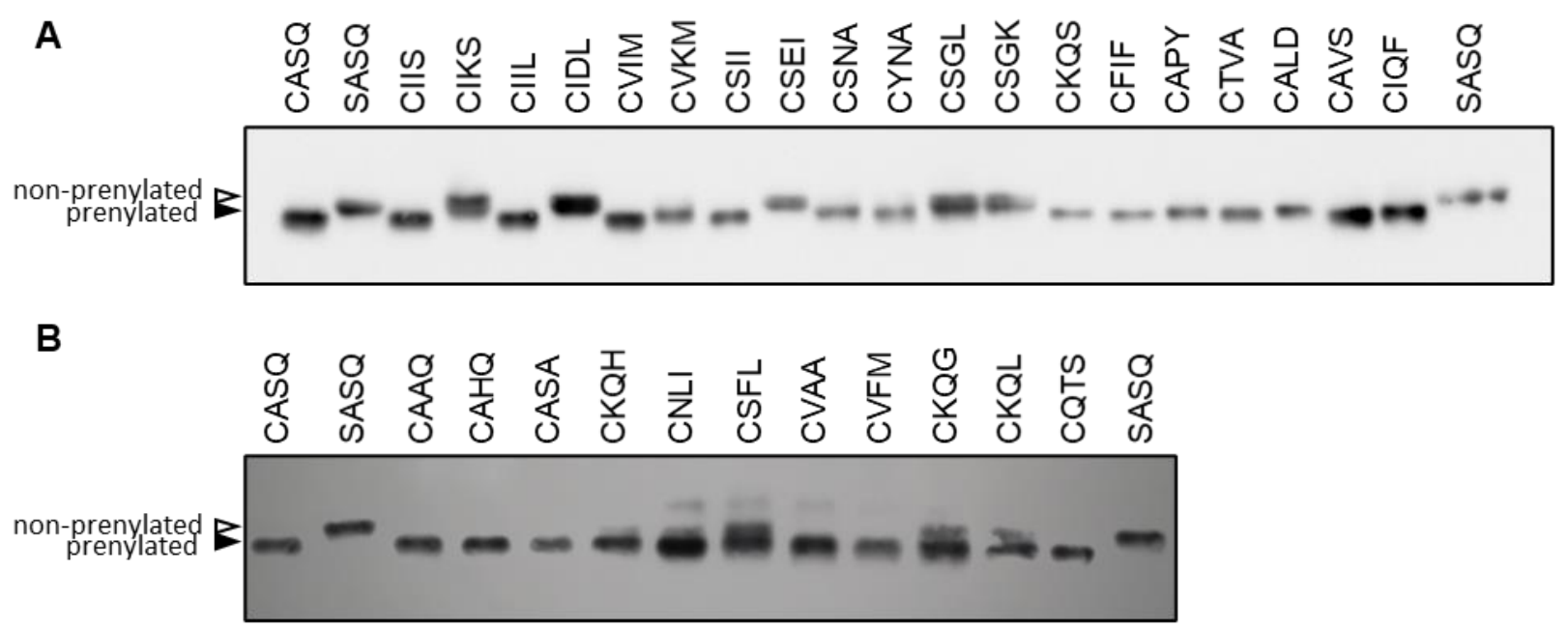

C

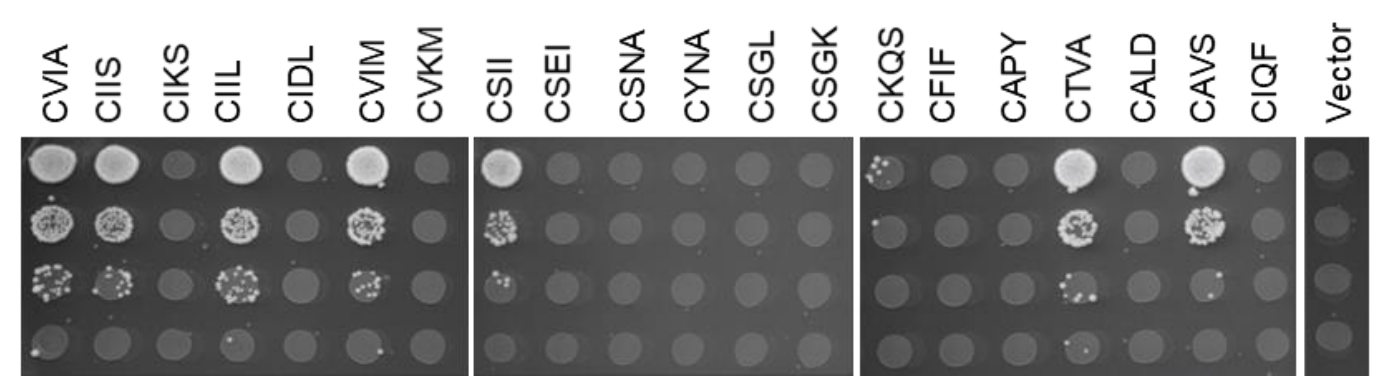

Fig 4. Empirically determined prenylation and cleavage of various Cxxx sequences. Yeast strains lacking chromosomally encoded YDJ1 (yWS304 or yWS2544, ydj1 MFA2 (SM2331, mfa1 $\Delta$ mfa2 $\Delta$ ) were engineered to individually express the indicated Ydj1p-Cxxx or a-factor-Cxxx variant, respectively, using a plasmid-based expression system (Table S2). A, B) Prenylation of the indicated naturally occurring Cxxx sequences in yeast $(A)$ or global Cxxx sequences (B) were determined by Ydj1p-gel shift assay. Yeast extracts were evaluated by SDS-PAGE and anti-Ydj1p immunoblot to reveal prenylated (closed triangle) and non-prenylated sequences (open triangle). Partial prenylation (i.e. doublet bands) were counted as a positive result. C) Cleavage of the indicated Cxxx sequences was determined by the a-factor mating assay. MATa yeast cultures were serial diluted 10-fold in the presence of excess MATa yeast (IH1793) and plated on SD media. Mating is indicated by diploid growth and is reported relative to mating exhibited by wildtype a-factor (CVIA).

For assessing cleavage, we used the yeast a-factor mating pheromone as a reporter (Fig 4B).

Canonical modification of a-factor (i.e., prenylation, cleavage, and carboxylmethylation) is required for mating of haploid yeast, which can be quantified as an indirect measure of a-factor production. As noted previously, for this assessment, we only evaluated the 14 sequences that 
were confirmed as being prenylated by Ydj1p gel-shift, regardless of whether they were predicted to be prenylated by any computational method. In this case, Freq outperformed SVMESM-1b, correctly predicting cleavage for $93 \%$ of sequences compared to $71 \%$, respectively; FBP and PrePS are not able to predict cleavage, so they were not evaluated (Table 3). For sequences where mating is observed, the mating levels are comparable to that of the wild type a-factor sequence (CVIA) (Fig 4B), indicative of complete rather than partial cleavage.

\section{Limitations of machine learning for predicting CaaX protein PTMs}

While SVM-ESM-1b can predict prenylation and cleavage, one limitation is that it does not provide any information about enzyme specificity due to the lack of enzyme-specific training information. For both prenylation and proteolysis, there are two possible enzymes for each reaction. For prenylation, FTase and GGTase-I can each prenylate a wide array of CaaX proteins with $\mathrm{C} 15$ farnesyl and C20 geranylgeranyl, respectively, while for proteolysis, Rce1p and Ste24p are both able to cleave the farnesylated CVIA motif of a-factor, but selectivity is observed for other motifs. The determinants of substrate specificity have not been fully ascertained for the aforementioned enzymes. A case in point is proteolysis of the CaaX motif CSIM, a sequence found on human prelamin A that has long thought to be a substrate of both CaaX proteases. SVM-ESM-1b and PSSM both predict that CSIM is cleaved, which we confirmed by using the a-factor reporter. When both proteases were present, comparable mating levels were observed between strains expressing a-factor in the context of the native CVIA motif that is cleaved by both Rce1p and Ste24p, the CTLM motif that is Rce1p-specific, and the CSIM motif (Fig 5A). When evaluated in the context of just one CaaX protease, we observed that all three motifs could be cleaved by Rce1p, but only CVIA was cleaved by Ste24p (Fig 5B). A similar result was observed when evaluating the human CaaX proteases in our yeast system (Fig 5C). Our observations are consistent with multiple reports challenging the 
role of Ste24p as an authentic CaaX protease, including a recent in vitro study demonstrating the inability of the human Ste24 ortholog, ZMPSTE24 to cleave at the Cys(farnesyl)-Ser bond of the CSIM motif, as would be expected for a CaaX protease (24).

A

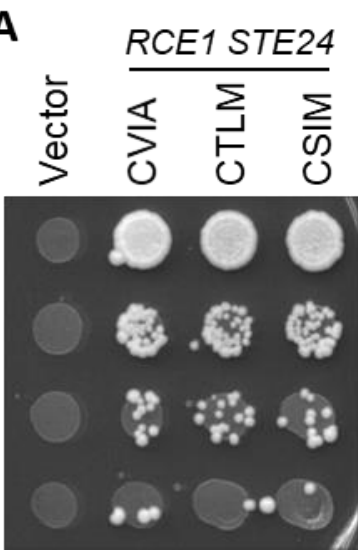

B

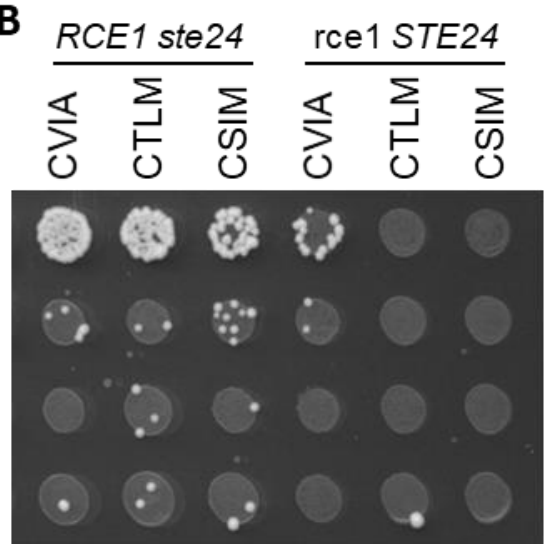

C

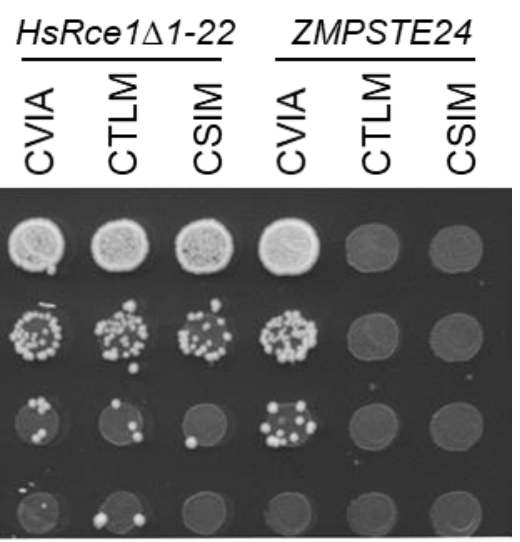

Fig 5. Rce1 is responsible for cleavage of yeast a-factor-CSIM. Yeast strains expressing the indicated a-factor Cxxx variant as the sole source of a-factor were evaluated as described for Fig 4 in the context of yeast and human CaaX proteases. Yeast strains expressing A) both yeast CaaX proteases (SM2331, $m f a 1 \Delta m f a 2 \Delta$ ), B) one or the other yeast CaaX protease (yWS2393, mfa1 $m$ fa2 $\Delta$ ste24; yWS2462, mfa1 $\mathrm{mfa} 2 \Delta$ rce 1), or C) plasmid-based human CaaX proteases (pWS130, HsRce1 11-22; pWS1609, ZMPSTE24) in a strain lacking both yeast

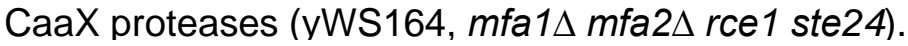

\section{Discussion}

A collection of in vivo, in silico and in vitro observations support a wider array of prenylation substrates than those previously defined by the $\mathrm{COOH}$-terminal CaaX motif $(8-12,21)$. Among the new substrates are those that lack aliphatic amino acids at the $\mathrm{a}_{1}$ and $\mathrm{a}_{2}$ position, leading to a broader definition for the prenylation motif. Using the machine learning platform SVM paired with ESM-1b training on CaaX motifs identified using both shunted and canonical reporters, we have developed a robust prediction algorithm for protein prenylation. SVM-based predictions suggest that approximately $33 \%$ of all $8000 \mathrm{Cxxx}$ motifs are prenylatable. This estimate is approximately $50 \%$ higher than the number of potential targets predicted by PrePS and is approximately double the number of sequences predicted by FlexPepBind (FPB). These 
findings are not meant to be indicative of the number of prenylated proteins in a cell since far fewer than all 8000 possible Cxxx motifs are encoded in genomes. For example, S. cerevisiae encodes only 89 proteins that end in Cxxx. Of these, SVM-ESM-1b predicted $46 \%(n=41)$ to be prenylated. By comparison, FPB and PrePS predicted $27 \%(n=24)$ and $32 \%(n=29)$ of yeast proteins to be prenylated, respectively. Confirmation of SVM-predicted prenylation will need to be evaluated on a case-by-case basis or by application of emerging methods for in vivo labeling of prenyl proteins to firmly establish whether SVM is an improvement over previous methods. We fully expect that the predicted SVM-based prenylation in some cases may not be possible in natural proteins due to inaccessibility of the $\mathrm{COOH}$ terminus to cytosolic prenyltransferases, either due to steric constraints for cytosolic proteins or the incompatible topology of membrane or secreted proteins. Despite the potential limitations of our prediction method, it is clear that SVM-ESM-1b predicted prenylation of known, non-canonical Cxxx sequences in instances where other methods did not (e.g., Ydj1p CASQ and Pex19p CKQQ), suggesting that SVM is an improvement for identifying prenylated proteins as a whole. Moreover, the non-canonical CKQS sequence associated with the histone chaperone Nap1p is also predicted to be prenylated by our SVM-ESM-1b model. To date, there exists no direct evidence for yeast Nap1p prenylation, but such evidence does exist for human and plant Nap1 homologs, which both possess a similar CKQQ motif $(25,26)$. Notably, the CKQQ sequence is also present on the human tumor suppressor Lkb1, another well documented prenylprotein (27).

As part of this study, we were also able to develop SVM-ESM-1b into a first-ever method for distinguishing between shunted (i.e., prenylation only) and cleaved sequences (i.e., canonical). Of the approximately 2600 sequences predicted to be prenylated by SVM, approximately $63 \%$ are predicted to be shunted and the remaining 37\% cleaved. Again, these findings are not meant to reflect the actual ratio of shunted and cleaved prenylated proteins in cells. In fact, we observe that the predictions are somewhat inversed within the yeast proteome. Of the 41 
sequences predicted to be prenylated, $27 \%$ are predicted to be shunted and the remaining $73 \%$ cleaved. This observation suggests that the cleavage and carboxymethylation of the prenylated $\mathrm{COOH}$ terminus may serve an important role in vivo, potentially increasing membrane association, as historically expected for canonical CaaX modifications. While the role of the isoprenyl group on shunted proteins remains unclear, we posit that this PTM may help mediate protein-protein interactions and/or provide a structural role rather than contribute to membrane association. This is supported by observations made on the human protein Spindly, whose Cxxx motif of CPQQ was predicted to be shunted by our SVM model, and for which a farnesyldependent protein complex interaction has been proposed $(28,29)$.

An unexpected result from this study was the observation that Freq and SVM-ESM-1b had a similar level of accuracy for prenylation prediction of the validation set $(77 \%$ and $80 \%$, respectively). As noted previously, Freq globally predicted more prenylated sequences than SVM-ESM-1b (42\% and 33\%, respectively), which is consistent with Freq having a higher false positive rate compared to SVM for our negative training set ( $40 \%$ and $3 \%$, respectively). This suggests to us that Freq overpredicts prenylation. It's also worth noting that while Freq and SVM-ESM1b rely on the same data set for predictions, their predictions are not coincident, indicating that predictions are fundamentally different for the two methods. Long term, we expect that future advancements in machine learning will lead to better prediction performance relative to the Freq-based method.

To further improve our prediction methods, one aspect that we wish to especially improve upon is the high false negative rate for prenylation predictions that was determined empirically by evaluating a small subset of test sequences $(n=31$; Table 5$)$. While a larger test set may yield a more accurate false negative rate, it remains possible that the high negative false rate is simply due to the training datasets themselves being too small or somehow compromised. We have 
high confidence that our positive prenylation training set is composed of prenylated sequences that, importantly, were derived from studies involving both canonical and shunted reporters. Our negative training test set, however, was derived from a single study that relied on a canonical reporter, and it is suspected that shunted sequences may be among the negative hits in that study, thus poisoning the quality of our negative test set. Our future studies are aimed at identifying a set of sequences that better reflect non-prenylatable sequences for use as an improved negative training set that we expect to lead to improved prenylation predictions and a lower false negative rate.

Interestingly, we observed that several models out-performed SVM-ESM-1b for cleavage prediction (e.g., PSSM, Freq). As previously noted, a larger set of test sequences may be needed to better assess performance. Alternatively, it may be that a better genetic test for cleavage is required. Previous studies have reported that geranylgeranylated a-factor has less mating activity in vivo $(18,21,30)$, suggesting that the genetic mating assay may only work well in the context of farnesylated a-factor. This potentially impacts results associated with the CFIF and CIQF sequences in our test set; the terminal Phe is a preferred GGTase-I feature. SVMESM-1b predicted prenylation of both sequences while SVM-ESM-1b, PSSM and Freq methods all predicted cleavage. Prenylation was confirmed in the context of Ydj1p, but neither sequence supported a-factor mating activity that would be indicative of cleavage. It remains unclear whether lack of mating activity is due to shunting or geranylgeranylation. Because of this issue, it is difficult to fully assess the accuracy of any of the cleavage predictors described in this study. In terms of the CaaX proteases, while CSIM was identified as a canonical motif, additional genetic studies utilizing a-factor were needed to resolve whether cleavage was mediated by Rce1p or Ste24p. As the yeast a-factor mating pheromone is the only known substrate of Ste24p to date, it is tempting to speculate that Rce1p is the main and possibly only 
relevant CaaX protease. If that eventually bears out to be the case, then our cleavage predictors could be used to infer Rce1p specificity.

Altogether, we have demonstrated that machine learning can be developed into a useful tool to prediction prenylation and cleavage events associated with CaaX proteins. The utility of this tool is reflected by its ability to better identify possible shunted sequences relative to other publicly available prediction methods, in addition to identifying canonically modified sequences. These findings represent an important step in expanding the full scope of prenylatable motifs in yeast. Given the high degree of target specificity exhibited by both prenyltransferases and CaaX proteases across species, it is likely that the prenylatable space identified by this study also represents the full scope of prenylated motifs in humans. Among these are sequences associated with proteins that represent potential new additions to the prenylome, which has implications for the impact of prenyltransferase and protease inhibitors being developed as therapeutics.

\section{Acknowledgements}

We thank Avrom Caplan (City College of New York) for anti-Ydj1p antibody, Patrick Casey (Duke University) for the baculovirus vector encoding human Rce1, and Ora Furman-Schueler (Hebrew University of Jerusalem) for sharing FlexPepBind scores. We also thank Jacob Greenway (Schmidt Lab, UGA) and members of the Schmidt Lab for their assistance with methods, reagent preparation, and critical discussions.

Funding: This work was supported by NIH funds to WKS and NK (NIH NIGMS GM132606, https://www.nih.gov/) and funds to WKS (NIH NIGMS R01GM117148, https://www.nih.gov/). The funders had no role in study design, data collection and analysis, decision to publish, or preparation of the manuscript. 
Competing interests: The authors have declared no competing interests exist.

\section{References}

1. Hartman HL, Hicks KA, Fierke CA. Peptide Specificity of Protein Prenyltransferases Is Determined Mainly by Reactivity Rather than Binding Affinity. Biochemistry. 2005;44(46):1531424.

2. Lane KT, Beese LS. Thematic review series: lipid posttranslational modifications. Structural biology of protein farnesyltransferase and geranylgeranyltransferase type I. Journal of lipid research. 2006;47(4):681-99.

3. Wang M, Casey PJ. Protein prenylation: unique fats make their mark on biology. Nature reviews Molecular cell biology. 2016;17(2):110-22.

4. Wright LP, Philips MR. Thematic review series: lipid posttranslational modifications. CAAX modification and membrane targeting of Ras. Journal of lipid research. 2006;47(5):88391.

5. Hampton SE, Dore TM, Schmidt WK. Rce1: mechanism and inhibition. Critical reviews in biochemistry and molecular biology. 2018:1-18.

6. Maurer-Stroh S, Eisenhaber F. Refinement and prediction of protein prenylation motifs. Genome biology. 62005. p. R55.

7. Maurer-Stroh S, Koranda M, Benetka W, Schneider G, Sirota FL, Eisenhaber F. Towards complete sets of farnesylated and geranylgeranylated proteins. PLoS computational biology. 2007;3(4):e66.

8. Stein V, Kubala MH, Steen J, Grimmond SM, Alexandrov K. Towards the Systematic Mapping and Engineering of the Protein Prenylation Machinery in Saccharomyces cerevisiae. PloS one. 2015;10(3):e0120716.

9. Hougland JL, Lamphear CL, Scott SA, Gibbs RA, Fierke CA. Context-dependent substrate recognition by protein farnesyltransferase. Biochemistry. 2009;48(8):1691-701. 10. Hougland JL, Hicks KA, Hartman HL, Kelly RA, Watt TJ, Fierke CA. Identification of novel peptide substrates for protein farnesyltransferase reveals two substrate classes with distinct sequence selectivities. Journal of molecular biology. 2010;395(1):176-90.

11. Reid TS, Terry KL, Casey PJ, Beese LS. Crystallographic analysis of CaaX prenyltransferases complexed with substrates defines rules of protein substrate selectivity. Journal of molecular biology. 2004;343(2):417-33.

12. London N, Lamphear CL, Hougland JL, Fierke CA, Schueler-Furman O. Identification of a novel class of farnesylation targets by structure-based modeling of binding specificity. PLoS computational biology. 2011;7(10):e1002170.

13. Sousa SF, Coimbra JT, Paramos D, Pinto R, Guimarães RS, Teixeira V, et al. Molecular dynamics analysis of a series of 22 potential farnesyltransferase substrates containing a CaaXmotif. Journal of molecular modeling. 2013;19(2):673-88.

14. DeGraw AJ, Palsuledesai C, Ochocki JD, Dozier JK, Lenevich S, Rashidian M, et al. Evaluation of alkyne-modified isoprenoids as chemical reporters of protein prenylation. Chemical biology \& drug design. 2010;76(6):460-71.

15. Onono FO, Morgan MA, Spielmann HP, Andres DA, Subramanian T, Ganser A, et al. A tagging-via-substrate approach to detect the farnesylated proteome using two-dimensional electrophoresis coupled with Western blotting. Molecular \& cellular proteomics : MCP. 2010;9(4):742-51.

16. Palsuledesai CC, Ochocki JD, Kuhns MM, Wang YC, Warmka JK, Chernick DS, et al. Metabolic Labeling with an Alkyne-modified Isoprenoid Analog Facilitates Imaging and Quantification of the Prenylome in Cells. ACS chemical biology. 2016;11(10):2820-8. 
17. Storck EM, Morales-Sanfrutos J, Serwa RA, Panyain N, Lanyon-Hogg T, Tolmachova T, et al. Dual chemical probes enable quantitative system-wide analysis of protein prenylation and prenylation dynamics. Nature Chemistry. 2019.

18. Trueblood CE, Boyartchuk VL, Picologlou EA, Rozema D, Poulter CD, Rine J. The CaaX proteases, Afc1p and Rce1p, have overlapping but distinct substrate specificities. Molecular and cellular biology. 2000;20(12):4381-92.

19. Hildebrandt ER, Cheng M, Zhao P, Kim JH, Wells L, Schmidt WK. A shunt pathway limits the CaaX processing of Hsp40 Ydj1p and regulates Ydj1p-dependent phenotypes. eLife. 2016;5.

20. Caplan AJ, Tsai J, Casey PJ, Douglas MG. Farnesylation of YDJ1p is required for function at elevated growth temperatures in Saccharomyces cerevisiae. The Journal of biological chemistry. 1992;267(26):18890-5.

21. Berger BM, Kim JH, Hildebrandt ER, Davis IC, Morgan MC, Hougland JL, et al. Protein Isoprenylation in Yeast Targets $\mathrm{COOH}-$ Terminal Sequences Not Adhering to the CaaX Consensus. Genetics. 2018;210(4):1301-16.

22. Stormo GD, Schneider TD, Gold L, Ehrenfeucht A. Use of the 'Perceptron' algorithm to distinguish translational initiation sites in E. coli. Nucleic Acids Res. 1982;10(9):2997-3011.

23. Platt J. Probabilistic outputs for support vector machines and comparisons to regularized likelihood methods. Advances in large margin classifiers. 1999;10(3):61-74.

24. Nie L, Spear E, Babatz TD, Quigley A, Dong YY, Chu A, et al. A new paradigm for Prelamin A proteolytic processing by ZMPSTE24: the upstream SY^LL cleavage occurs first and there is no CaaX processing by ZMPSTE24. bioRxiv. 2020:2020.05.13.093849.

25. Kho Y, Kim SC, Jiang C, Barma D, Kwon SW, Cheng J, et al. A tagging-via-substrate technology for detection and proteomics of farnesylated proteins. Proceedings of the National Academy of Sciences of the United States of America. 2004;101(34):12479-84.

26. Galichet A, Gruissem W. Developmentally controlled farnesylation modulates AtNAP1;1 function in cell proliferation and cell expansion during Arabidopsis leaf development. Plant Physiol. 2006;142(4):1412-26.

27. Collins SP, Reoma JL, Gamm DM, Uhler MD. LKB1, a novel serine/threonine protein kinase and potential tumour suppressor, is phosphorylated by cAMP-dependent protein kinase (PKA) and prenylated in vivo. The Biochemical journal. 2000;345 Pt 3(Pt 3):673-80.

28. Moudgil DK, Westcott N, Famulski JK, Patel K, Macdonald D, Hang H, et al. A novel role of farnesylation in targeting a mitotic checkpoint protein, human Spindly, to kinetochores. The Journal of cell biology. 2015;208(7):881-96.

29. Mosalaganti S, Keller J, Altenfeld A, Winzker M, Rombaut P, Saur M, et al. Structure of the RZZ complex and molecular basis of its interaction with Spindly. The Journal of cell biology. 2017;216(4):961-81.

30. Caldwell GA, Wang SH, Naider F, Becker JM. Consequences of altered isoprenylation targets on a-factor export and bioactivity. Proceedings of the National Academy of Sciences of the United States of America. 1994;91(4):1275-9.

31. Kawashima S, Pokarowski P, Pokarowska M, Kolinski A, Katayama T, Kanehisa M. AAindex: amino acid index database, progress report 2008. Nucleic Acids Res. 2008;36(Database issue):D202-5.

32. Rives A, Meier J, Sercu T, Goyal S, Lin Z, Liu J, et al. Biological structure and function emerge from scaling unsupervised learning to 250 million protein sequences. Proceedings of the National Academy of Sciences. 2021;118(15).

33. Tipping ME, Bishop CM. Mixtures of Probabilistic Principal Component Analyzers.

Neural Computation. 1999;11(2):443-82.

34. Henikoff S, Henikoff JG. Amino acid substitution matrices from protein blocks.

Proceedings of the National Academy of Sciences of the United States of America.

1992;89(22):10915-9. 
35. Pedregosa F, Varoquaux G, Gramfort A, Michel V, Thirion B, Grisel O, et al. Scikit-learn: Machine Learning in Python. J Mach Learn Res. 2011;12(null):2825-30.

36. Harris CR, Millman KJ, van der Walt SJ, Gommers R, Virtanen P, Cournapeau D, et al. Array programming with NumPy. Nature. 2020;585(7825):357-62.

37. Paszke A, Gross S, Massa F, Lerer A, Bradbury J, Chanan G, et al. PyTorch: An Imperative Style, High-Performance Deep Learning Library2019 December 01, 2019:[arXiv:1912.01703 p.]. Available from: https://ui.adsabs.harvard.edu/abs/2019arXiv191201703P.

38. Hunter JD. Matplotlib: A 2D Graphics Environment. Computing in Science and Engg. 2007;9(3):90-5.

39. Waskom ML. Seaborn: statistical data visualization. Journal of Open Source Software. $2021 ; 6(60): 3021$.

40. Crooks GE, Hon G, Chandonia JM, Brenner SE. WebLogo: a sequence logo generator. Genome research. 2004;14(6):1188-90.

41. Elble R. A simple and efficient procedure for transformation of yeasts. BioTechniques. 1992;13(1):18-20.

42. Plummer LJ, Hildebrandt ER, Porter SB, Rogers VA, McCracken J, Schmidt WK. Mutational analysis of the ras converting enzyme reveals a requirement for glutamate and histidine residues. The Journal of biological chemistry. 2006;281(8):4596-605.

43. Giaever G, Chu AM, Ni L, Connelly C, Riles L, Véronneau S, et al. Functional profiling of the Saccharomyces cerevisiae genome. Nature. 2002;418(6896):387-91.

44. Oldenburg KR, Vo KT, Michaelis S, Paddon C. Recombination-mediated PCR-directed plasmid construction in vivo in yeast. Nucleic Acids Res. 1997;25(2):451-2.

45. Cadiñanos J, Schmidt WK, Fueyo A, Varela I, López-Otín C, Freije JMP. Identification, functional expression and enzymic analysis of two distinct CaaX proteases from Caenorhabditis elegans. The Biochemical journal. 3702003. p. 1047-54.

46. Hildebrandt ER, Arachea BT, Wiener MC, Schmidt WK. Ste24p Mediates Proteolysis of Both Isoprenylated and Non-prenylated Oligopeptides. The Journal of biological chemistry. 2016;291(27):14185-98.

47. Kim S, Lapham AN, Freedman CG, Reed TL, Schmidt WK. Yeast as a tractable genetic system for functional studies of the insulin-degrading enzyme. The Journal of biological chemistry. 2005;280(30):27481-90.

48. Shoemaker DD, Lashkari DA, Morris D, Mittmann M, Davis RW. Quantitative phenotypic analysis of yeast deletion mutants using a highly parallel molecular bar-coding strategy. Nature genetics. 1996;14(4):450-6.

49. Michaelis S, Herskowitz I. The a-factor pheromone of Saccharomyces cerevisiae is essential for mating. Molecular and cellular biology. 1988;8(3):1309-18.

50. Chen P, Sapperstein SK, Choi JD, Michaelis S. Biogenesis of the Saccharomyces cerevisiae mating pheromone a-factor. The Journal of cell biology. 1997;136(2):251-69.

51. Sikorski RS, Hieter P. A system of shuttle vectors and yeast host strains designed for efficient manipulation of DNA in Saccharomyces cerevisiae. Genetics. 1989;122(1):19-27.

52. Krishnankutty RK, Kukday SS, Castleberry AJ, Breevoort SR, Schmidt WK. Proteolytic processing of certain CaaX motifs can occur in the absence of the Rce1p and Ste24p CaaX proteases. Yeast (Chichester, England). 2009;26(8):451-63. 


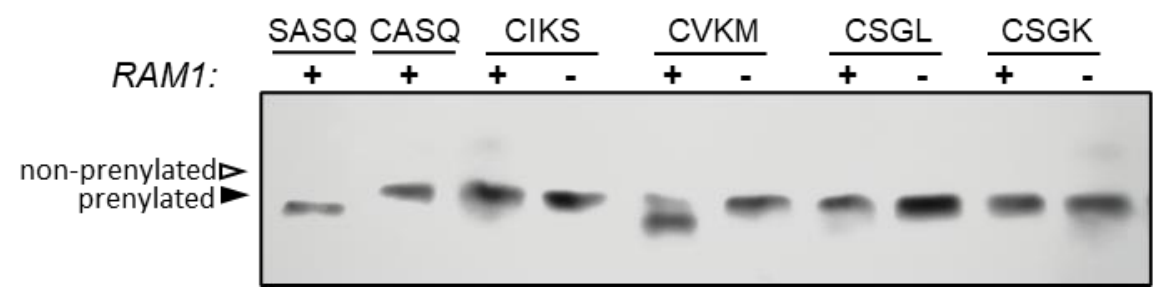

Fig S1. Confirmation of prenylation status on ambiguous $\mathrm{Cxxx}$ sequences. Yeast strains lacking chromosomally encoded YDJ1 +/- RAM1 (yWS304, ydj1 $\Delta$ or yWS2542, ydj1 $1 \Delta$ ram1 $1 \Delta$ ) expressing Ydj1p-Cxxx plasmids of sequences were evaluated in the presence/absence of FTase (RAM1 gene) as described in Fig 4. Sequences were selected from Fig 4A for further evaluation due to unclear gel shift or prenylation status.

Supplementary Table S1. Probability estimates and prediction calls for prenylation and cleavage of naturally occurring yeast Cxxx sequences as reported by the SVM-ESM-1b model.

\begin{tabular}{|c|c|c|c|c|c|}
\hline \multirow[b]{2}{*}{ yeast protein } & \multirow[b]{2}{*}{ motif } & \multicolumn{2}{|c|}{ prenylation } & \multicolumn{2}{|c|}{ cleavage } \\
\hline & & score $^{a}$ & prediction & score & prediction \\
\hline Ras2 & CIIS & 0.9969 & + & 6.1594 & + \\
\hline Hmg1 & CIKS & 0.2587 & - & NA & NA \\
\hline Rho2 & CIIL & 0.9880 & + & 5.0875 & + \\
\hline Ssp2 & CIDL & 0.0534 & - & NA & NA \\
\hline Skt5, MiY1 & CVIM & 1.0000 & + & 8.9377 & + \\
\hline Tbs1 & CVKM & 0.1811 & - b & 1.8026 & - \\
\hline YDL022C-A & CSII & 0.9762 & + & 5.9957 & + \\
\hline YBR096W & CSEI & 0.1155 & - & NA & NA \\
\hline YMR265C & CSNA & 0.1339 & - b & -0.6976 & - \\
\hline Pet18 & CYNA & 0.0738 & $-b$ & -0.2218 & - \\
\hline Lih1 & CSGL & 0.1847 & - & NA & NA \\
\hline Cup1 & CSGK & 0.0034 & - & NA & NA \\
\hline Nap1 & CKQS & 0.5680 & + & 1.8671 & - \\
\hline Cst26 & CFIF & 0.9800 & + & 5.1732 & $t^{b}$ \\
\hline YIL134C-A & CAPY & 0.6833 & + & -1.2698 & - \\
\hline Atr1 & CTVA & 0.9860 & + & 4.8219 & + \\
\hline Las21 & CALD & 0.6017 & + & 2.4882 & - \\
\hline YDL009C & CAVS & 0.9831 & + & 4.3306 & + \\
\hline Sua5 & CIQF & 0.9018 & + & 4.0671 & - \\
\hline
\end{tabular}

aProbability estimates were determined for the SVM-ESM-1b model using Platt-scaling. Signs represent predictions of prenylation and that were reported as positive (+) or negative (-) by the SVM-ESM-1b model. NA - not applicable.

brediction differs from empirical observation. 
Supplementary Table S2. Prediction calls for cleavage of naturally occurring yeast Cxxx sequences by indicated model.

\begin{tabular}{|c|c|c|c|c|}
\hline yeast protein & motif & PSSMa,b & Naïve Bayes & kNN \\
\hline Ras2 & CIIS & + & + & + \\
\hline Hmg1 & CIKS & NA & NA & NA \\
\hline Rho2 & CIIL & + & + & + \\
\hline Ssp2 & CIDL & NA & NA & NA \\
\hline Skt5, MiY1 & CVIM & + & + & + \\
\hline Tbs1 & CVKM & - & - & - \\
\hline YDL022C-A & CSII & + & + & + \\
\hline YBR096W & CSEI & NA & NA & NA \\
\hline YMR265C & CSNA & - & - & - \\
\hline Pet18 & CYNA & - & - & - \\
\hline Lih1 & CSGL & NA & NA & NA \\
\hline Cup1 & CSGK & NA & NA & NA \\
\hline Nap1 & CKQS & - & - & - \\
\hline Cst26 & CFIF & $+^{\mathrm{c}}$ & $t^{\mathrm{c}}$ & $+^{\mathrm{c}}$ \\
\hline YIL134C-A & CAPY & - & - & - \\
\hline Atr1 & CTVA & + & + & + \\
\hline Las21 & CALD & - & - & - \\
\hline YDL009C & CAVS & $-c$ & $-\mathrm{c}$ & $-\mathrm{c}$ \\
\hline Sua5 & CIQF & - & - & - \\
\hline \multirow{2}{*}{\multicolumn{2}{|c|}{$\begin{array}{r}\text { number observed/predicted } \\
\% \text { observed/predicted }\end{array}$}} & $12 / 14$ & $12 / 14$ & $12 / 14$ \\
\hline & & 85.7 & 85.7 & 85.7 \\
\hline \multicolumn{2}{|c|}{ number false positive } & $1 / 7$ & $1 / 7$ & $1 / 7$ \\
\hline \multicolumn{2}{|c|}{$\%$ false positive } & 14.3 & 14.3 & 14.3 \\
\hline \multicolumn{2}{|c|}{ number false negative } & $1 / 7$ & $1 / 7$ & $1 / 7$ \\
\hline \multicolumn{2}{|c|}{$\%$ false negative } & 14.3 & 14.3 & 14.3 \\
\hline
\end{tabular}

aPSSM - PSSM sequence; Naïve Bayes - Naïve Bayes AAindex; kNN - kNN sequence.

bSigns represent predictions of cleavage that were reported as positive (+) or negative (-) by the indicated model. NA - not applicable.

'Prediction differs from empirical observation. 
Supplementary Table S3. Yeast strains used in this study.

\begin{tabular}{|c|c|c|}
\hline Strain & Genotype & Reference \\
\hline BY4741 & MATa his $3 \Delta 1$ leu $2 \Delta 0$ met $15 \Delta 0$ ura $3 \Delta 0$ & $(48)$ \\
\hline $\begin{array}{l}\text { IH1793; } \\
\text { ATCC\#204279 }\end{array}$ & MATa lys 1 & $(49)$ \\
\hline SM2331 & MATa trp1 leu2 ura3 his4 can $1 \mathrm{mfa} 1-\Delta 1 \mathrm{mfa} 2-\Delta 1$ & $(50)$ \\
\hline yWS164 & $\begin{array}{l}\text { MATa trp1 leu2 ura3 his4 can1 mfa1- } \triangle 1 \text { mfa2- } \Delta 1 \\
\text { rce1::TRP1 ste24::KANR }\end{array}$ & $(45)$ \\
\hline yWS304 & MATa his $3 \Delta 1$ leu2 $\Delta 0$ met15 150 ura3 $\Delta 0$ ydj1 $1 \Delta:: K A N^{R}$ & $(43)$ \\
\hline yWS2393 & $\begin{array}{l}\text { MATa trp1 leu2 ura3 his4 can1 mfa1- } \Delta 1 \text { mfa2- } \Delta 1 \\
\text { ste24::KANR }\end{array}$ & This study \\
\hline yWS2462 & $\begin{array}{l}\text { MATa trp1 leu2 ura3 his4 can1 mfa1- } \Delta 1 \text { mfa2- } \Delta 1 \\
\text { rce1::KAN }\end{array}$ & This study \\
\hline yWS2542 & $\begin{array}{l}\text { MATa his3 leu2 met15 ura3 ydj1 } \triangle:: N A T^{R} \\
\text { ram1 } 1:: K A N^{R}\end{array}$ & $(21)$ \\
\hline yWS2544 & MATa his $3 \Delta 1$ leu2 $\Delta 0$ met15 10 ura3 $\Delta 0$ ydj1 $\Delta:: N A T^{R}$ & $(21)$ \\
\hline
\end{tabular}


Supplementary Table S4. Yeast expression plasmids used in this study

\begin{tabular}{|c|c|c|c|}
\hline Gene & Identifier & Genotype & Reference \\
\hline \multirow[t]{3}{*}{ vectors } & pRS315 & CEN LEU2 & (51) \\
\hline & pRS316 & CEN URA3 & (51) \\
\hline & pRS415 & CEN LEU2 & (51) \\
\hline HsRce1 & pWS130 & $2 \mu$ URA3 $P_{P G K} H s R c e 1 \triangle 22$ & This study \\
\hline HsSte24 & pWS1609 & CEN URA3 $P_{P G K} H s S T E 24$ & This study \\
\hline \multirow{33}{*}{ YDJ1 } & pWS942 & CEN URA3 YDJ1 & (19) \\
\hline & pWS1132 & CEN URA3 YDJ1-SASQ & (19) \\
\hline & pWS1343 & CEN URA3 YDJ1-CASA & (21) \\
\hline & pWS1372 & CEN URA3 YDJ1-CAAQ & (21) \\
\hline & pWS1410 & CEN URA3 YDJ1-CVAA & This study \\
\hline & pWS1411 & CEN URA3 YDJ1-CKQS & This study \\
\hline & pWS1437 & CEN URA3 YDJ1-CAHQ & This study \\
\hline & pWS1456 & CEN URA3 YDJ1-CAKS & This study \\
\hline & pWS1460 & CEN URA3 YDJ1-CQTS & This study \\
\hline & pWS1461 & CEN URA3 YDJ1-CSFL & This study \\
\hline & pWS1463 & CEN URA3 YDJ1-CVIM & This study \\
\hline & pWS1729 & CEN URA3 YDJ1-CTDS & This study \\
\hline & pWS1745 & CEN URA3 YDJ1-CALD & This study \\
\hline & pWS1746 & CEN URA3 YDJ1-CAPY & This study \\
\hline & pWS1747 & CEN URA3 YDJ1-CAVS & This study \\
\hline & pWS1748 & CEN URA3 YDJ1-CFIF & This study \\
\hline & pWS1749 & CEN URA3 YDJ1-CIDL & This study \\
\hline & pWS1751 & CEN URA3 YDJ1-CIIL & This study \\
\hline & pWS1752 & CEN URA3 YDJ1-CIKS & This study \\
\hline & pWS1753 & CEN URA3 YDJ1-CIQF & This study \\
\hline & pWS1757 & CEN URA3 YDJ1-CSEI & This study \\
\hline & pWS1758 & CEN URA3 YDJ1-CSGK & This study \\
\hline & pWS1759 & CEN URA3 YDJ1-CSGL & This study \\
\hline & pWS1760 & CEN URA3 YDJ1-CSII & This study \\
\hline & pWS1761 & CEN URA3 YDJ1-CSNA & This study \\
\hline & pWS1762 & CEN URA3 YDJ1-CTVA & This study \\
\hline & pWS1763 & CEN URA3 YDJ1-CVKM & This study \\
\hline & pWS1764 & CEN URA3 YDJ1-CYNA & This study \\
\hline & pWS1830 & CEN URA3 YDJ1-CNLI & This study \\
\hline & pWS1834 & CEN URA3 YDJ1-CVFM & This study \\
\hline & pWS2021 & CEN URA3 YDJ1-CKQG & This study \\
\hline & pWS2022 & CEN URA3 YDJ1-CKQH & This study \\
\hline & pWS2025 & CEN URA3 YDJ1-CKQL & This study \\
\hline \multirow[t]{14}{*}{ MFA1 } & pWS610 & CEN LEU2 MFA1 & (52) \\
\hline & pWS613 & CEN LEU2 MFA1-CTLM & (19) \\
\hline & pWS846 & CEN LEU2 MFA1-CKQS & (52) \\
\hline & pWS1561 & CEN LEU2 MFA1-CSIM & This study \\
\hline & pWS1562 & CEN LEU2 MFA1-CIIS & This study \\
\hline & pWS1671 & CEN LEU2 MFA1-CTVA & This study \\
\hline & pWS1730 & CEN LEU2 MFA1-CALD & This study \\
\hline & pWS1733 & CEN LEU2 MFA1-CIQF & This study \\
\hline & pWS1734 & CEN LEU2 MFA1-CYNA & This study \\
\hline & pWS1738 & CEN LEU2 MFA1-CVIM & This study \\
\hline & pWS1739 & CEN LEU2 MFA1-CSGL & This study \\
\hline & pWS1777 & CEN LEU2 MFA1-CAVS & This study \\
\hline & pWS1778 & CEN LEU2 MFA1-CFIF & This study \\
\hline & pWS1779 & CEN LEU2 MFA1-CIIL & This study \\
\hline
\end{tabular}


bioRxiv preprint doi: https://doi.org/10.1101/2021.11.30.470454; this version posted November 30, 2021. The copyright holder for this preprint (which was not certified by peer review) is the author/funder. All rights reserved. No reuse allowed without permission.

pWS1780

pWS1781

pWS1782

pWS1783

pWS1784

pWS1785

pWS1788

pWS1809
CEN LEU2 MFA1-CIKS

CEN LEU2 MFA1-CIDL CEN LEU2 MFA1-CSII CEN LEU2 MFA1-CSEI CEN LEU2 MFA1-CSNA CEN LEU2 MFA1-CAPY CEN LEU2 MFA1-CSGK CEN LEU2 MFA1-CVKM
This study

This study

This study

This study

This study

This study

This study

This study 
Supplementary Table S5. PCR Oligonucleotides used in this study.

\begin{tabular}{|c|c|c|c|}
\hline Gene & Oligo & Mutation & Sequence (5' to 3') \\
\hline $\begin{array}{l}\text { Plasmid } \\
\text { UTR }\end{array}$ & oWS219a & $N A^{b}$ & tgaCCATGATTACGCCAAGC \\
\hline \multirow[t]{15}{*}{ YDJ1 } & oWS999 & CVAA & $\begin{array}{l}\text { TCCGATGAAGAAGAACAAGGTGGCGAAGGTGTTCAATGT } \\
\text { GTTGCCGCATGAttttcttgataaaaaaagatca }\end{array}$ \\
\hline & oWS1000 & CKQS & $\begin{array}{l}\text { TCCGATGAAGAAGAACAAGGTGGCGAAGGTGTTCAATGT } \\
\text { AAGCAGAGCTGAttttcttgataaaaaaagatca }\end{array}$ \\
\hline & oWS1008 & $\mathrm{CAHQ}$ & $\begin{array}{l}\text { GATTCCGATGAAGAAGAACAAGGTGGCGAAGGTGTTCAA } \\
\text { TGTGCTCAtCAATGAttttcttgataaaaaaagatcaac }\end{array}$ \\
\hline & oWS1028 & CIIS & $\begin{array}{l}\text { GATTCCGATGAAGAAGAACAAGGTGGCGAAGGTGTTCAA } \\
\text { TGTatcatttctTGAttttcttgataaaaaaagatca }\end{array}$ \\
\hline & oWS1032 & CQTS & $\begin{array}{l}\text { GATTCCGATGAAGAAGAACAAGGTGGCGAAGGTGTTCAA } \\
\text { TGTcaaacatctTGAttttcttgataaaaaaagatca }\end{array}$ \\
\hline & oWS1033 & CSFL & $\begin{array}{l}\text { GATTCCGATGAAGAAGAACAAGGTGGCGAAGGTGTTCAA } \\
\text { TGTtctttttgTGAttttcttgataaaaaaagatca }\end{array}$ \\
\hline & oWS1035 & CVIM & $\begin{array}{l}\text { GATTCCGATGAAGAAGAACAAGGTGGCGAAGGTGTTCAA } \\
\text { TGTgttatcatgTGAttttcttgataaaaaaagatca }\end{array}$ \\
\hline & oWS1051 & $N A^{b}$ & GGTATGAAGTGGAGGGAGGAT \\
\hline & oWS1334 & CALD & $\begin{array}{l}\text { TCCGATGAAGAAGAACAAGGTGGCGAAGGTGTTCAATGT } \\
\text { GCTTTGGATTGAttttcttgataaaaaaagatc }\end{array}$ \\
\hline & oWS1335 & CAPY & $\begin{array}{l}\text { TCCGATGAAGAAGAACAAGGTGGCGAAGGTGTTCAATGT } \\
\text { GCTCCATATTGAttttcttgataaaaaaagatc }\end{array}$ \\
\hline & oWS1336 & CAVS & $\begin{array}{l}\text { TCCGATGAAGAAGAACAAGGTGGCGAAGGTGTTCAATGT } \\
\text { GCTGTTTCTTGAtttcttgataaaaaaagatc }\end{array}$ \\
\hline & oWS1337 & CFIF & $\begin{array}{l}\text { TCCGATGAAGAAGAACAAGGTGGCGAAGGTGTTCAATGT } \\
\text { TTTATTTTTTGAttttcttgataaaaaaagatc }\end{array}$ \\
\hline & oWS1338 & CIDL & $\begin{array}{l}\text { TCCGATGAAGAAGAACAAGGTGGCGAAGGTGTTCAATGT } \\
\text { ATTGATTTGTGAttttcttgataaaaaaagatc }\end{array}$ \\
\hline & oWS1340 & CIIL & $\begin{array}{l}\text { TCCGATGAAGAAGAACAAGGTGGCGAAGGTGTTCAATGT } \\
\text { ATTATTTTGTGAttttcttgataaaaaaagatc }\end{array}$ \\
\hline & oWS1341 & CIKS & $\begin{array}{l}\text { TCCGATGAAGAAGAACAAGGTGGCGAAGGTGTTCAATGT } \\
\text { ATTAAATCTTGAttttcttgataaaaaaagatc }\end{array}$ \\
\hline
\end{tabular}




\begin{tabular}{|c|c|c|c|}
\hline & oWS1342 & CIQF & $\begin{array}{l}\text { TCCGATGAAGAAGAACAAGGTGGCGAAGGTGTTCAATGT } \\
\text { ATTCAATTTTGAttttcttgataaaaaaagatc }\end{array}$ \\
\hline & oWS1346 & CSEI & $\begin{array}{l}\text { TCCGATGAAGAAGAACAAGGTGGCGAAGGTGTTCAATGT } \\
\text { TCTGAAATTTGAttttcttgataaaaaaagatc }\end{array}$ \\
\hline & oWS1347 & CSGK & $\begin{array}{l}\text { TCCGATGAAGAAGAACAAGGTGGCGAAGGTGTTCAATGT } \\
\text { TCTGGTAAATGAttttcttgataaaaaaagatc }\end{array}$ \\
\hline & oWS1348 & CSGL & $\begin{array}{l}\text { TCCGATGAAGAAGAACAAGGTGGCGAAGGTGTTCAATGT } \\
\text { TCTGGTTTGTGAttttcttgataaaaaaagatc }\end{array}$ \\
\hline & oWS1349 & CSII & $\begin{array}{l}\text { TCCGATGAAGAAGAACAAGGTGGCGAAGGTGTTCAATGT } \\
\text { TCTATTATTTGAttttcttgataaaaaaagatc }\end{array}$ \\
\hline & oWS1350 & CSNA & $\begin{array}{l}\text { TCCGATGAAGAAGAACAAGGTGGCGAAGGTGTTCAATGT } \\
\text { TCTAATGCTTGAttttcttgataaaaaaagatc }\end{array}$ \\
\hline & oWS1351 & CTVA & $\begin{array}{l}\text { TCCGATGAAGAAGAACAAGGTGGCGAAGGTGTTCAATGT } \\
\text { ACTGTTGCTTGAtttcttgataaaaaaagatc }\end{array}$ \\
\hline & oWS1352 & CVKM & $\begin{array}{l}\text { TCCGATGAAGAAGAACAAGGTGGCGAAGGTGTTCAATGT } \\
\text { GTTAAAATGTGAttttcttgataaaaaaagatc }\end{array}$ \\
\hline & oWS1353 & CYNA & $\begin{array}{l}\text { TCCGATGAAGAAGAACAAGGTGGCGAAGGTGTTCAATGT } \\
\text { TATAATGCTTGAttttcttgataaaaaaagatc }\end{array}$ \\
\hline & oWS1423 & CNLI & $\begin{array}{l}\text { TCCGATGAAGAAGAACAAGGTGGCGAAGGTGTTCAATGT } \\
\text { AATTTGATTTGAttttcttgataaaaaaagatc }\end{array}$ \\
\hline & oWS1444 & CVFM & $\begin{array}{l}\text { TCCGATGAAGAAGAACAAGGTGGCGAAGGTGTTCAATGT } \\
\text { GTTTTTATGTGAtttcttgataaaaaaagatc }\end{array}$ \\
\hline & oWS1583 & CKQG & $\begin{array}{l}\text { tccgatgaagaagaacaaggtggcgaaggtgttcaatgtAAACAAGGTtgattt } \\
\text { tcttgataaaaaaagatc }\end{array}$ \\
\hline & oWS1584 & $\mathrm{CKQH}$ & $\begin{array}{l}\text { tccgatgaagaagaacaaggtggcgaaggtgttcaatgtAAACAACATtgattt } \\
\text { tcttgataaaaaaagatc }\end{array}$ \\
\hline & oWS1587 & CKQL & $\begin{array}{l}\text { tccgatgaagaagaacaaggtggcgaaggtgttcaatgtAAACAATTGtgattt } \\
\text { tcttgataaaaaaagatc }\end{array}$ \\
\hline MFA1 & oWS356 & CVKM & $\begin{array}{l}\text { AACTATATTATCAAAGGTGTCTTCTGGGACCCAGCATGcgt } \\
\text { aaaaatgTAGTTTCTGCGTACAAAAACGCGT }\end{array}$ \\
\hline & oWS1178 & CSIM & $\begin{array}{l}\text { AACTATATTATCAAAGGTGTCTTCTGGGACCCAGCATGctct } \\
\text { atcatgTAGTTTCTGCGTACAAAAACGCGT }\end{array}$ \\
\hline & oWS1179 & CIIS & $\begin{array}{l}\text { AACTATATTATCAAAGGTGTCTTCTGGGACCCAGCATGCat } \\
\text { catttctTAGTTTCTGCGTACAAAAACGCGT }\end{array}$ \\
\hline
\end{tabular}




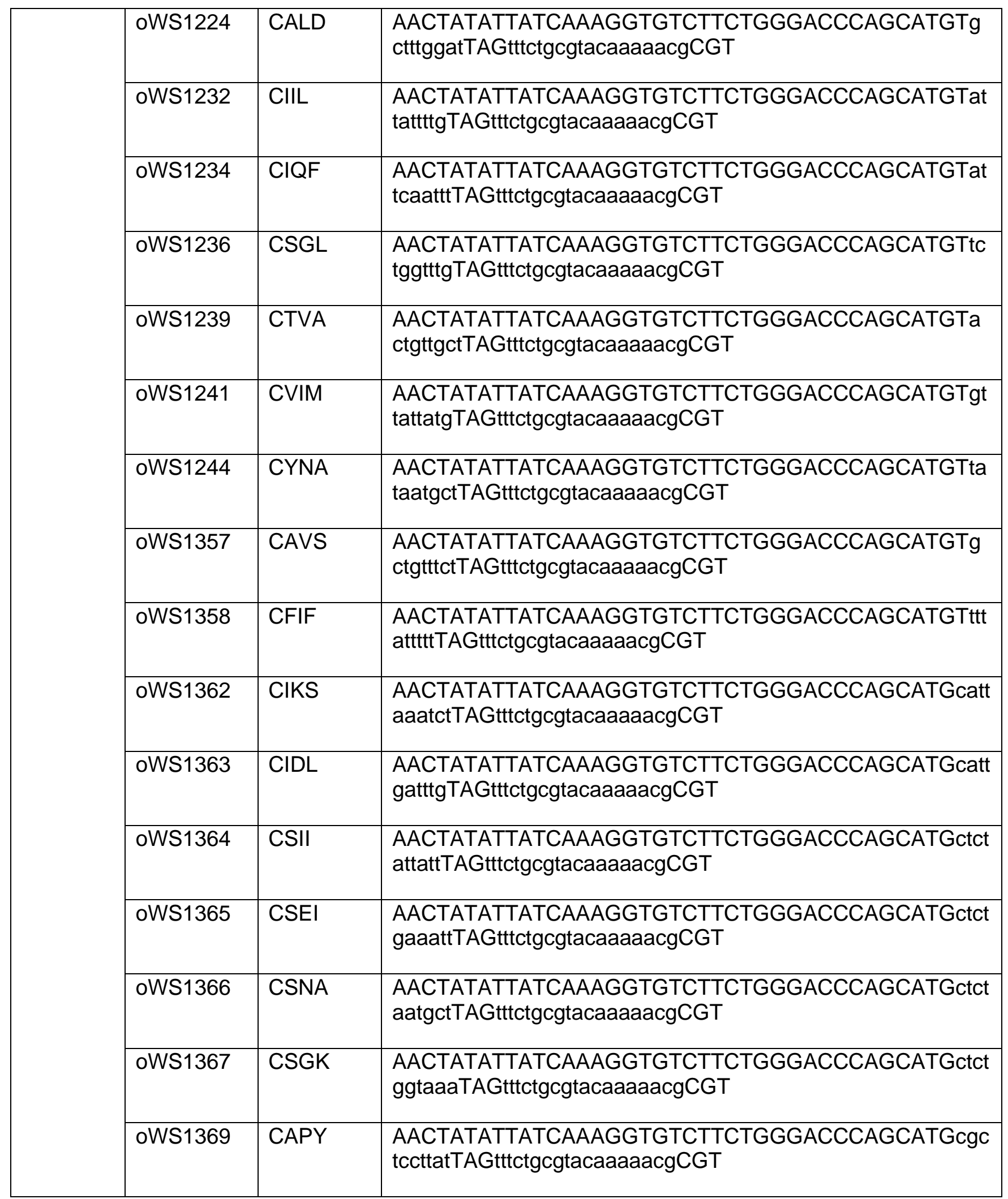

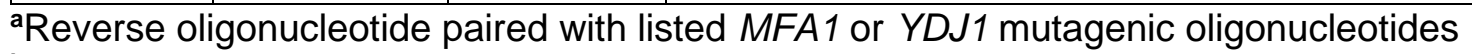

bNot applicable.

'Lowercase letters indicate differences from wildtype sequences. 